\title{
Changes in Mitochondrial Function Resulting from Synaptic Activity in the Rat Hippocampal Slice
}

\author{
Vytautas P. Bindokas, ${ }^{1}$ Chong C. Lee, ${ }^{1}$ William F. Colmers, ${ }^{2}$ and Richard J. Miller ${ }^{1}$ \\ ${ }^{1}$ Department of Pharmacological and Physiological Sciences, University of Chicago, Chicago, Illinois 60637, and \\ 2Department of Pharmacology, University of Alberta, Edmonton, Canada
}

\begin{abstract}
Digital imaging microfluorimetry was used to visualize changes in mitochondrial potential and intracellular $\mathrm{Ca}^{2+}$ concentration, $\left[\mathrm{Ca}^{2+}\right]_{\mathrm{i}}$, in thick slices of rat hippocampus. Electrical activity, especially stimulus train-induced bursting (STIB) activity, produced slow, prolonged changes in mitochondrial potential within hippocampal slices as revealed by fluorescence measurements with rhodamine dyes. Changes in mitochondrial potential showed both temporal and spatial correlations with the intensity of the electrical activity. Patterned changes in mitochondrial potential were observed to last from tens of seconds to minutes as the consequence of epileptiform discharges. STIB-associated elevations in $\left[\mathrm{Ca}^{2+}\right]_{i}$ were also pro-
\end{abstract}

Synaptic activity has metabolic consequences beyond the obvious need for neurons to restore the ionic equilibria perturbed by the activation of synaptic conductances. Among other effects, synaptic events can regulate the activity of proteins involved in signal transduction and even the expression of proteins whose genes are induced as a result of synaptic signaling (e.g., Ghosh and Greenberg, 1995). Synaptically induced metabolic processes can be observed using various methods, yielding images that reflect the pattern and intensity of synaptic activity. Examples range from static images of activity-induced glucose uptake in monkey striate cortex (Hubel et al., 1978) to millisecond-resolution images of intracellular $\mathrm{Ca}^{2+}$ concentration $\left(\left[\mathrm{Ca}^{2+}\right]_{\mathrm{i}}\right)$ dynamics in dendritic spines (Denk et al., 1996). Altered intrinsic optical properties may also arise from activity-dependent increases in tissue perfusion and/or local swelling. Thus, light reflectance is decreased in electrically active regions of in vivo primate visual cortex (Blasdel and Salama, 1986; Ts'o et al., 1990), increased light transmittance accompanies spreading depression in hippocampal slices (Andrew and MacVicar, 1994), and similar correlations of electrical activity with optical properties are seen in isolated guinea pig brain (Federico et al., 1994).

Mitochondria are critical for the regulation of ATP, $\left[\mathrm{Ca}^{2+}\right]_{\mathrm{i}}$, and other important intracellular signals. Approximately $90 \%$ of

\footnotetext{
Received Jan. 12, 1998; revised March 31, 1998; accepted April 3, 1998.

This work was supported by National Institutes of Health Grants DA-02575, DA-02121, MH-40165, DK-42086, DK-44840, and NS-33502 to R.J.M. and by Grant MT10520 from the Medical Research Council of Canada to W.F.C., who is also a Medical Scientist of Alberta Heritage Foundation for Medical Research. C.C.L. was supported by National Institutes of Health Grants 5T32GM07151-22 and HD07009. We thank Dr. W. A. Wilson (Duke University) for helpful comments and discussions regarding some of the data and Dr. S. Lindquist (University of Chicago) for allowing us to use the confocal microscope.

Correspondence should be addressed to Dr. Richard J. Miller, Department of Pharmacological and Physiological Sciences, University of Chicago, 947 East 58th Street, Chicago IL 60637.

Copyright (ㄷ) 1998 Society for Neuroscience $\quad 0270-6474 / 98 / 184570-18 \$ 05.00 / 0$
}

longed and exhibited a spatial pattern similar to that of the mitochondrial depolarization. The mitochondrial depolarization was sensitive to TTX and glutamate receptor blockers $\left(\left[\mathrm{Mg}^{2+}\right]\right.$ 。 and CNQX or DNQX plus D-AP-5) and to the inhibition of glutamate release by activation of presynaptic NPY receptors. The monitoring of mitochondrial potential in slice preparations provides a new tool for mapping synaptic activity in the brain and for determining the roles of mitochondria in regulation of brain synaptic activity.

Key words: rhodamine 123; TMRM; TMRE; mitochondria; epilepsy; rhod-2; neuropeptide Y; peptide YY; synaptic plasticity; STIB
ATP generated in the brain occurs by oxidative phosphorylation within mitochondria (cf. Gunter et al., 1994). Neurons and glia require ATP to establish and maintain ionic gradients and for other energy-dependent processes common to most cells. Neurons expend considerable energy on the buffering, sequestration, and extrusion of $\mathrm{Ca}^{2+}$ to maintain a low $\left[\mathrm{Ca}^{2+}\right]_{\mathrm{i}}$. Mitochondria actively take up $\mathrm{Ca}^{2+}$ (McCormack et al., 1990), responding differentially to phasic versus static changes in $\left[\mathrm{Ca}^{2+}\right]_{i}$ (Sparagna et al., 1994; Hajnóczky et al., 1995). Mitochondrial dysfunction has been implicated in a variety of neurodegenerative states. Mitochondrial demise is an early, critical step in excitotoxicity (Ankarcrona et al., 1995; Khodorov et al., 1996; Schinder et al., 1996; White and Reynolds, 1996). They also play a prominent role in apoptotic death (Kroemer et al., 1997). Additionally, mitochondria produce reactive oxygen species (Dugan et al., 1995; Reynolds and Hastings, 1995; Bindokas et al., 1996) and may also be targets for these agents (e.g., Castro et al., 1994; Hausladen and Fridovich, 1994; Schweizer and Richter, 1994).

Epileptic seizures, remarkable for their duration and synchrony, rely on glutamatergic interconnections between hippocampal neurons of area CA3 (Traub and Miles, 1991), and NMDA receptor activation is important for seizure induction (e.g., Anderson et al., 1987; Stasheff et al., 1989). The excessive metabolic demands of seizure activity may damage mitochondria (Meldrum, 1986) and may produce cell death by excitotoxic mechanisms (e.g., Olney et al., 1986). Mitochondrial dysfunction may also be causally associated with certain epilepsies (e.g., Shoffner et al., 1990; Torbergsen et al., 1991). An interaction between changes in mitochondrial functional state and the control of synaptic activity has also been recently described at peripheral synapses (Nguyen and Atwood, 1994; Tang and Zucker, 1997).

To investigate this relationship, we have determined the effects of synaptic events on mitochondrial potential in hippocampal 
slices. We observed changes in rhod-2 fluorescence $\left(\left[\mathrm{Ca}^{2+}\right]_{\mathrm{i}}\right)$ and rhodamine dye fluorescence (mitochondrial polarization) using an in vitro model of epileptiform activity in which reduced $\left[\mathrm{Mg}^{2+}\right]_{\mathrm{o}}$ and electrical stimulation produced stimulus traininduced bursting activity (STIB) (Stasheff et al., 1985, 1989). Our results show that the imaging of mitochondrial potential can reveal previously undetected physiological activity and provide a new tool for studying of the roles of mitochondria in the regulation of synaptic behavior.

\section{MATERIALS AND METHODS}

Slice preparations. Horizontal slices of hippocampi (500-600 $\mu \mathrm{m}$ thick) were made from male Sprague Dawley rats aged 17-24 d old, similar to Klapstein and Colmers (1997). After decapitation and rapid brain removal, the hippocampi were quickly microdissected, glued down leaning vertically against agar blocks in a small chamber, submerged in cold, oxygenated artificial CSF (aCSF), and sectioned in the transverse plane using a vibratome (TPI, St. Louis, MO). Slices were allowed to equilibrate at room temperature for at least $1 \mathrm{hr}$ before use in experiments. The aCSF contained (in mM): $127 \mathrm{NaCl}, 3 \mathrm{KCl}, 2 \mathrm{MgSO}_{4}, 2 \mathrm{CaCl}_{2}, 1.23$ $\mathrm{NaH}_{2} \mathrm{PO}_{4}$ ), $25 \mathrm{NaHCO}_{3}$, and 10 glucose and was bubbled with $5 \%$ $\mathrm{CO}_{2} / 95 \% \mathrm{O}_{2}$. Electrical activity was enhanced by use of low- $\mathrm{Mg}^{2+}$ aCSF containing $0.9 \mathrm{mM} \mathrm{Mg}^{2+}$ (Stasheff et al., 1989).

Drug solution osmolarity (305 mOsm) was balanced to within 3-5 mOsm for all solutions. Peptide YY (PYY; American Peptide) and tetrodotoxin (TTX; Sigma, St. Louis, MO) solutions, both at $1 \mu \mathrm{M}$, were freshly prepared in aCSF from concentrated stocks. 6,7Dinitroquinoxaline-2,3-dione (DNQX; $10 \mu \mathrm{M}$; Research Biochemicals, Natick, MA) and 2-amino-5-phosphonopentanoic acid (D-AP-5; 100 $\mu \mathrm{M}$; Research Biochemicals) were made from aqueous stocks; 6-cyano7-nitroquinoxaline-2,3-dione (CNQX; $10 \mu \mathrm{M}$; Research Biochemicals) was diluted from a $1000 \times$ stock in DMSO.

Dye loading. Slices were usually preloaded with indicator dyes before microfluorescence measurements by maintaining them in small containers containing dye solution in bubbled aCSF at $23^{\circ} \mathrm{C}$. Mitochondria were stained in aCSF containing $10 \mu \mathrm{g} / \mathrm{ml}$ (or $26 \mu \mathrm{M}$ ) freshly prepared rhodamine 123 (R123; diluted from a $1000 \times$ stock in 95\% EtOH; Molecular Probes, Eugene, OR) for at least $30 \mathrm{~min}$. Because R123 staining was reasonably stable, the superfusion salines used in experiments did not contain R123. The lack of R123 replenishment, although significantly reducing background fluorescence during experiments, did decrease our ability to detect mitochondrial hyperpolarizations. Some slices were loaded with tetramethyl rhodamine methyl ester (TMRM; 1 $\mu \mathrm{M})$ and kept in solutions containing $100 \mathrm{~nm}$ TMRM. Other slices were loaded with tetramethyl rhodamine ethyl ester (TMRE; $1 \mu \mathrm{M}$ ) or loaded with the potential-insensitive dye $10-N$-nonyl acridine orange (NAO; 1 $\mu \mathrm{M})$. For measurements of $\left[\mathrm{Ca}^{2+}\right]_{\mathrm{i}}$, slices were loaded with rhod-2 AM (4.3 $\mu \mathrm{M}$; Molecular Probes) for $30 \mathrm{~min}$.

Slices were transferred to a glass coverslip that formed the bottom of an open chamber ( $1 \mathrm{ml}$ of total volume) on the stage of an inverted microscope (Nikon Diaphot) and held in place by means of silver weights. Superfusate was fed by gravity $(1 \mathrm{ml} / \mathrm{min})$ and heated to $33-35^{\circ} \mathrm{C}$. Electrical stimulation was applied with a stainless steel bipolar electrode inserted into stratum radiatum in the $\mathrm{CA} 2 / \mathrm{CA} 3$ region of the slice. Stimulus trains were generated by a Grass stimulator (S8800) equipped with a stimulus isolation unit. Stimulation consisted of trains of four stimuli (0.1 msec; $20-35 \mathrm{~V}$; repeated at $100 \mathrm{~Hz}$ ) repeated at $200 \mathrm{msec}$ intervals for $6-17$ trains. Stimuli were applied no $<10 \mathrm{~min}$ apart to increase chances of eliciting STIB responses. Extracellular field potentials were recorded via a patch pipette (5-10 $\mathrm{M} \Omega$ resistance) filled with low- $\mathrm{Mg}^{2+} \mathrm{aCSF}$ and placed in the CA3c s. pyramidale. The recording electrode was connected to the headstage of an Axopatch 1D amplifier in current-clamp mode. The electrical signal was monitored with pCLAMP 5.5 (Axon Instruments, Foster City, CA), and data were digitized at 50 $\mathrm{kHz}$ and also stored on videotape (Instrutech VR-10A Digital Data Recorder). Taped records were played back into FETCHEX and analyzed with CLAMPFIT or custom routines in MATLAB 4.2 (MathWorks Inc., Natick, MA).

Microfluorimetry and image analyses. Fluorescence images were obtained on a Diaphot microscope (Nikon) using an EF numerical aperture (NA) $0.12,4 \times$ objective (Leitz) with epi-illumination by an attenuated (neutral density, 1.5) $150 \mathrm{~W}$ xenon arc lamp (Oriel Corporation, Stratford, CT), shutter (Metaltek, Raleigh, NC), and rhodamine filters (exci- tation, $510-560 \mathrm{~nm}$; dichromatic mirror, $580 \mathrm{~nm}$; emission > $590 \mathrm{~nm}$; Nikon) and an ICCD video camera (Hamamatsu C2400) under the control of MetaMorph software (Universal Imaging Corporation, West Chester, PA). Rhodamine optics were used with R123 to minimize the signal from slice autofluorescence. TMRM, TMRE, and rhod-2 fluorescence was also measured under these optical conditions. Some experiments were conducted using a $40 \times$, NA 0.75 long-working distance objective (Zeiss) or a $100 \times$, NA 1.3 (Leitz) objective. For some of the high-magnification studies, the excitation area was decreased by closing the field iris to minimize flare from tissue. Images (eight bit $=256$ gray levels) were averages of $64-128$ video frames to increase the signal-tonoise ratio and required $\sim 2-4 \mathrm{sec}$ per image acquisition. Stacks of 30 consecutive images $(\sim 1-2$ min total duration) were collected per image series and saved to disk. Camera and intensifier gains were not altered during an experiment. This resulted in some loss of signal-to-noise ratio because R123 signal intensity diminished with time.

Because changes in mitochondrial potential were commonly determined from images consisting of 128 video frame averages (collection time $=4.2 \mathrm{sec}$ per image), this typically limited temporal resolution to windows of $\sim 8.5 \mathrm{sec}$ (the difference between two images). However, observed fluorescence changes often lasted for many tens of seconds, and thus the temporal resolution was adequate to detect changes but may have aliased the peak responses. Some data were collected at twice the rate, but decreased signal-to-noise ratio was evident in image differences. Although these data were similar, the higher time resolution resulted in smaller incremental changes that made event detection actually somewhat more difficult.

Image stacks were later analyzed by a variety of methods to reveal spatial and temporal changes in fluorescence. Changes in fluorescence were calculated on a pixel-by-pixel basis (244 kilopixels/image). Analyses included calculation of the intensity change relative to the first image (relative fluorescence changes; $F_{n}-F_{1}$ ) and changes between adjacent temporal images [pairwise changes; $F_{n}-F_{(n-1)}$ ]), as well as calculation of SD or mean derivative for all or part of an image stack. A constant offset was added to each intensity value before subtraction to aid in detection of fluorescence decreases. The relative fluorescence change $\left(F_{n}-F_{1}\right)$ method revealed overall intensity changes over the duration of the data stack relative to the first image ("background") and is similar to that used for intrinsic signal analyses (MacVicar and Hochman, 1991; Andrew and MacVicar, 1994). The pairwise subtractions $\left[F_{n}-F_{(n-1)}\right]$ revealed trends limited to the interval required for collection of two images $(\sim 4$ or $8 \mathrm{sec})$ and are less sensitive to longer-term changes in fluorescence. These pairwise subtractions yield images containing the first derivative trends. Pixel-by-pixel variability calculations conducted on subsets of raw data images revealed the spatial distribution of any change and its magnitude (as in SD plots) as well as its direction in mean derivative plots. Both the relative and pairwise changes were obtained using routines within the MetaMorph software package. Variability matrices were calculated by a custom routine written in MATLAB. SDs over the extent of the image stack or a subset (6-30 images) were calculated for each pixel (positions $x_{1}$ to $x_{512}, y_{1}$ to $y_{480}$ across image to image $\left.{ }_{n}\right)$. For example, the $\operatorname{SD}\left(x_{1}, y_{1}\right)=\mathrm{SD}$ of intensities at $\left\{\left(x_{1}, y_{1}\right)\right.$ image $_{1} \ldots\left(x_{1}, y_{1}\right)$ image $\left._{n}\right]$. Mean derivative calculations determined the average of intensity differences between image pairs within the analysis stack on a pixel-by-pixel basis. For example, the mean derivative at pixel $x_{1}, y_{1}=$ mean $\left\{\left[\left(x_{1}, y_{1}\right)\right.\right.$ image $_{2}-\left(x_{1}, y_{1}\right)$ image $\left._{1}\right] \ldots\left[\left(x_{1}, y_{1}\right)\right.$ image $_{n}-$ $\left(x_{1}, y_{1}\right)$ image $\left.\left._{(n-1)}\right]\right\}$. Mean derivatives could be positive (net increasing fluorescence over time), zero (no change), or negative (net decrease over time) per pixel. The magnitude of the SDs and the derivative output matrices were converted into indexed gray scale images to reveal the spatially registered changes across the image stack acquisition time windows. These "variance" images were displayed after linear histogram equalization to common limits to increase contrast and could be falsecolored to better visualize trends. All images of a particular experiment were scaled identically. Some plots were further displayed as threedimensional intensity (mesh) plots using both height and color to code fluorescence changes by means of a custom MATLAB routine. Average raw fluorescence intensity plots for the entire slice or from selected regions of interest were created using MetaMorph.

No single data reduction method described above was able to convey all of the dynamic changes contained in the data sets. Minute as well as ordered changes were best revealed by time-lapsed movies created from image stacks or from the analyzed output images. Movie loops were played at three frames per sec (fps) and $15 \mathrm{fps}$ (time compression for 128 frame average images is $\sim 12.7 \times$ and $63.5 \times$, respectively). We have 

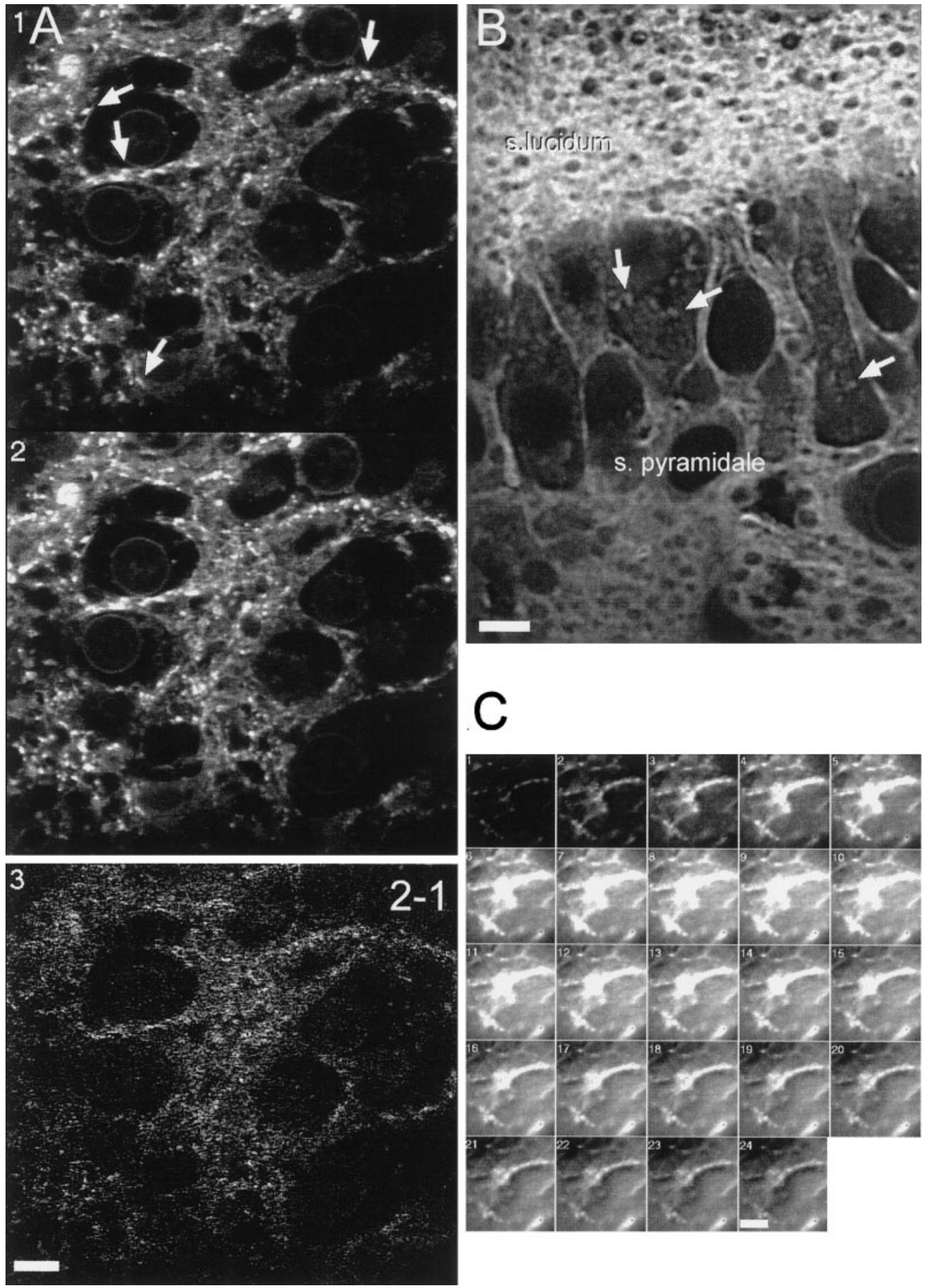

Figure 1. Rhodamine dyes stain mitochondria within thick hippocampal slices. Staining slices with rhodamine dyes results in a punctate staining pattern that is similar for R123, TMRM, and TMRE. A, Scanning laser confocal images of CA pyramidal neurons that were double stained with TMRE and NAO, a potential-insensitive dye, reveal that dyes are localized within mitochondrion-like organelles (e.g., arrows) that are especially abundant in the neuropil surrounding the neuronal somata. The NAO signal was colocalized to the TMRE compartments (data not shown); however, NAO also weakly stained other cellular membranes such as the nuclear envelope as is faintly evident in these images. This nonmitochondrial signal is absent in slices stained only with TMRM, TMRE, and R123 (e.g., B). A2 shows a second scan at this optical plane $\sim 15$ sec later and during the occurrence of a spontaneous wave of change in fluorescence. The pixel-by-pixel digital subtraction of $A 2-A 1$ shown in $A 3$ reveals that this spontaneously occurring increase in TMRE fluorescence is primarily restricted to the vicinity of the brightly stained organelles. $B$, A confocal image of a slice stained with R123 is shown. Mitochondria are present within the somata of CA3 pyramidal neurons (e.g., arrows). The s. lucidum (top) is strongly stained, indicating the presence of many mitochondria and/or mitochondria with greater transmembrane potentials. $C$, A TMRE-stained slice viewed at high magnification under conventional fluorescence optics and the spontaneously occurring changes in fluorescence over time are shown. These images, taken every 4 sec, are unprocessed data. Brightly stained organelles appear to outline the pyramidal neuronal somata (darker voids). The fluorescence increases first appear in the mitochondria and then spread to other cell regions. Weak oscillations are evident in some mitochondria, but these details are best observed in digital movies available in the electronic edition of this paper (http://www.jneurosci.org/supplemental/18/12/4570). Scale bars: $A, B, 5 \mu \mathrm{m} ; C, 10 \mu \mathrm{m}$. 
presented static montages of these image stacks, but they only convey the gross changes. Image stacks were converted to AVI movies or to animated GIFs. Inclusion of the time-lapse data in this paper is available as digital movies within the electronic version of this paper in the Journal of Neuroscience available to each subscriber (http://www.jneurosci.org/supplemental/18/12/4570). These movies are played back at $3 \mathrm{fps}$ except title and notation frames $(1 \mathrm{~Hz})$, and most movies represent an original time span of $\sim 127 \mathrm{sec}(2.1 \mathrm{~min})$.

Confocal microscopy. Dye distribution within some slices was examined with a Zeiss LSM 410 inverted confocal microscope. Slices were stained as above but were observed in HEPES-buffered saline (in mM): 144 $\mathrm{NaCl}, 10 \mathrm{HEPES}, 2 \mathrm{CaCl}_{2}, 1 \mathrm{MgCl}_{2}, 3 \mathrm{KCl}$, and $10 \mathrm{D}$-glucose, 306 mOsm, and pH $7.4(\mathrm{NaOH})$ because of lack of ability to gas the aCSF. Slices were examined using the 488 or $514 \mathrm{~nm}$ excitation lines with a $100 \times$, NA 1.2 objective or the $40 \times$ objective and a pinhole setting of $6-20$. Laser intensity was attenuated $10-30 \times$ to minimize bleaching. Line averaging (four or eight) was used in some images to improve the signal-to-noise ratio. Serial optical sections were obtained in the $z$-axis, or a single plane was rescanned at intervals of 8 or $15 \mathrm{sec}$. Dimensions of stained organelles in images from hippocampal slices and cultured primary hippocampal pyramidal neurons (12-18 d in vitro) were measured using MetaMorph software.

Abbreviations for anatomical structures used in the text are: s.oriens, stratum oriens; s.pyr., stratum pyramidale; s.luc., stratum lucidum; s.rad., stratum radiatum; DG, dentate gyrus; and EC, entorhinal cortex.

\section{RESULTS}

Data were collected from over 70 preparations for mitochondrial potential and seven preparations for determination of $\left[\mathrm{Ca}^{2+}\right]_{i}$.

\section{Changes in mitochondrial potential}

Rhodamine dyes are cationic, lipophilic dyes that selectively stain mitochondria in proportion to their highly negative transmembrane potential. Changes in transmembrane potential result in an altered ability of mitochondria to retain dye, with consequent changes in dye fluorescence intensity and distribution. Although R123 exhibits some self-quenching and binding to mitochondrial contents, it has been successfully used to record changes in mitochondrial potential in rat neurons (Duchen, 1992). Single loadings of slices with R123 allowed us to observe mitochondrial potential for at least several hours, depending on the particular experiment. Brief treatments that lead to mitochondrial depolarization released trapped dye from mitochondria into the cytosol and increased slice fluorescence. Mitochondria then appeared to recover dye with only a small net loss from the cells (see below), in agreement with measurements from isolated mitochondria (e.g., Lötscher et al., 1980) and similar to the situation observed in cultured neurons in vitro (Bindokas and Miller, 1995).

Slices loaded fairly uniformly with R123, but the prominent cell somata of the DG and s.pyr. of areas CA1 and CA3 formed distinct dark bands (see Figs. 1, 2, 3A). This may be because of lower mitochondrial densities (see below; Nafstad and Blackstad, 1966) and/or less-polarized mitochondrial potentials in somata versus the dendrites (Overly et al., 1996). Some of the most intense staining commonly occurred in the s.luc. (e.g., see Fig. $1 B)$. Loading invariably labeled mitochondria in both neurons and glia. The present low-magnification imaging approach did not permit us to assess separately the contribution of glial mitochondria to the events described below.

Rhodamine dyes were localized to mitochondria as assessed by the punctate staining pattern within neurons observed at high magnification (Fig. 1). Most brightly stained organelles remained in fixed locations within the slice, but a small population were seen to be transported within somata and cellular processes. Random movements were observed within 11 somata of pyramidal neurons (three of seven slices), and directed transport-like motion was seen in one case. The cellular structures were present within the somata of CA pyramidal neurons (Fig. 1B, e.g., arrows) but were more numerous and more brightly stained in the surrounding neuropil, especially the s. lucidum. These structures were detectable with conventional fluorescence microscopy (Fig. $1 C$ ) but best resolved with confocal laser microscopy (Fig. 1A,B). The fluorescence intensity in the vicinity of these organelles was dynamic, increasing and decreasing over time (Fig. $1 A, C$ ), sometimes in repeated oscillations (data not shown). The recurrence of oscillations suggests that the fluorescence changes were not caused by photodamage. The fluorescence staining pattern was identical for three different potential-sensitive dyes (R123, TMRM, and TMRE) at both the macroscopic and subcellular levels. Stained organelles within slices had dimensions that were statistically similar to those in cultured primary hippocampal neurons (slice average length, $0.66 \pm 0.06 \mu \mathrm{m}$; average breadth, $0.39 \pm 0.02 \mu \mathrm{m}$; in vitro, $0.79 \pm 0.03$ and $0.34 \pm 0.01 \mu \mathrm{m} ; p=0.23$ and 0.14 , respectively). The mitochondria-preferring, potentialinsensitive dye NAO (cf. Petit et al., 1994) likewise generated similar staining patterns at the macroscopic level as well as at high magnification (data not shown). However, NAO also faintly stained other cellular membranes such as the nuclear envelope (see Fig. 1A).

Behavior of the rhodamine signal from hippocampal slices conformed to expectations after treatments with drugs that specifically altered mitochondrial potential (Fig. 2). Oligomycin (10 $\mu \mathrm{M})$ blocks ATP synthase and leads to a mitochondrial hyperpolarization. For R123, this resulted in a decrease in average slice fluorescence, similar to that described in isolated neurons (Duchen, 1992). Similarly, succinate $(5 \mathrm{~mm})$ produced a slight decrease in R123 fluorescence, consistent with its ability to hyperpolarize the mitochondria (data not shown). In contrast, agents that depolarize mitochondria by blocking electron transport, such as antimycin $\mathrm{A}(\mathrm{AA} ; 10 \mu \mathrm{M})$, or the protonophore carbonyl cyanide p-trifluoromethoxyhydrazone (FCCP; $1 \mu \mathrm{M}$ ) produced an increase in average slice fluorescence (Fig. 2) in accordance with reports from isolated neurons (Duchen, 1992). Overall, oligomycin decreased average slice fluorescence by $5.7 \pm$ $1.7 \%(n=8)$, AA subsequently increased fluorescence $6.3 \pm$ $2.6 \%(n=7)$, and FCCP increased fluorescence $10.7 \pm 2.5 \%$ $(n=5)$. Because (1) potentiometric rhodamine dyes were sequestered within cellular organelles of mitochondrial appearance within neuronal somata as well as the neuropil and (2) changes in this signal after treatments with well-defined actions on mitochondrial potential corresponded to predicted changes in isolated mitochondrial and isolated neurons, it therefore seems that the fluorescence signals observed here in thick brain tissue slices likewise arise from changes in mitochondrial potential.

The R123 fluorescence was dependent on electrical activity within the slice. Figure $3 A$ shows the mean fluorescence for the average of six images obtained before stimulation and is representative of seven similar experiments. Figure $3 B$ shows the average of six images (4.2 sec each) taken during and after electrical stimulation with a seven-burst train of impulses that induced STIB activity (see inset in Fig. 3E). The digital difference of the two averages is scaled and shown in Figure $3 C$. The mean increase in fluorescence over the entire slice was 3.3 F1.U. This increase, however, was nonuniform, with most of the increase limited to the s. pyr., s.rad., and s.luc. in the CA3,2,1c regions as well as some increase in the s. oriens. The two dark regions in the s.luc. mark the location of the stimulation electrodes (see also Fig. $3 A, S$ ). Note also the large increase in a band from the EC (Fig. $3 A$, top right) through the 


\section{A}

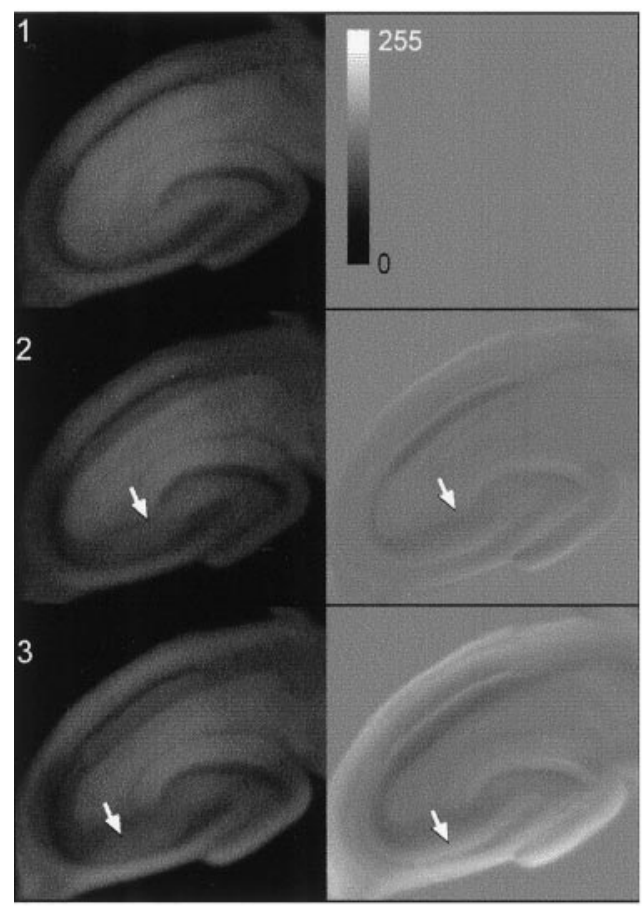

B

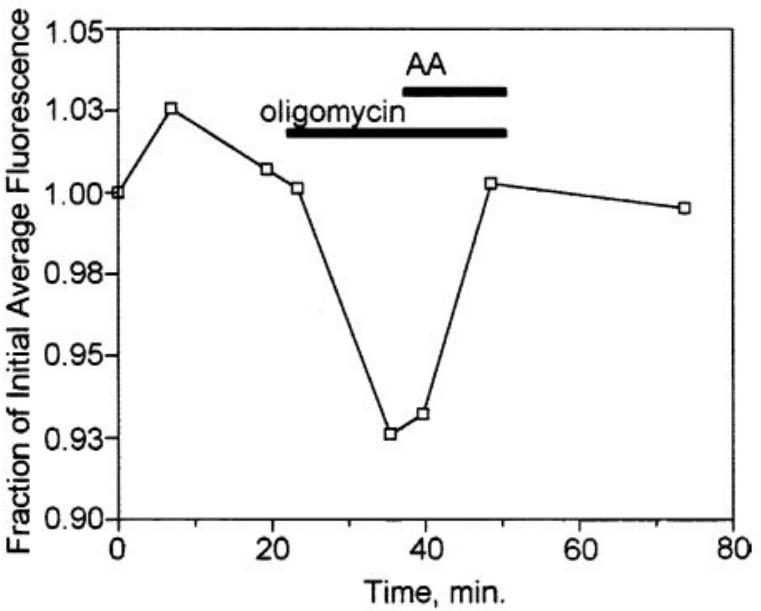

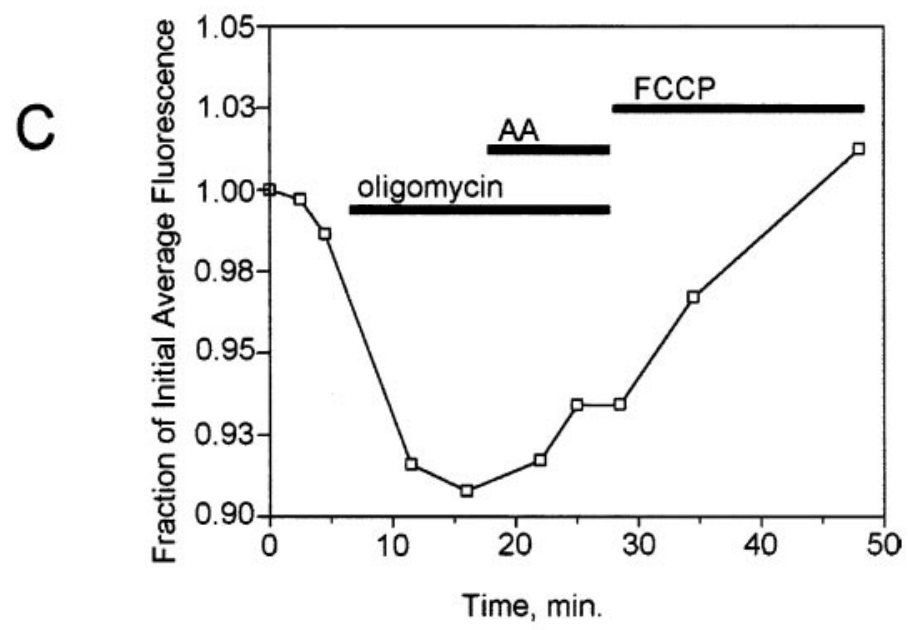

Figure 2. Fluorescence signals from hippocampal slices stained with R123 conform to expectations for mitochondrial hyper- and depolarizations. $A$, Left, The raw fluorescence images from a slice stained with R123 before $(A 1)$ and after $(A 2)$ treatment with the F1-F0 ATP synthase inhibitor oligomycin $(10 \mu \mathrm{M})$ and after the later addition of the electron transport chain inhibitor AA $(10 \mu \mathrm{M})(A 3)$ are shown. Right, The digital subtraction of the first image from the other images (here a constant offset of 150 was added to visualize signal decreases, and no other contrast manipulations were performed), indicating where fluorescence changes occurred, is shown. Oligomycin produced a decrease in overall fluorescence, especially in the CA3 region (arrows), consistent with a mitochondrial hyperpolarization. AA increased fluorescence (e.g., arrows) consistent with its ability to depolarize mitochondria. $B$, The full time course for this experiment is shown. Average fluorescence over the entire slice was normalized to the initial value. The average change in fluorescence was $-7 \%$ in oligomycin and was followed by full recovery in AA in this example. Durations of treatments are indicated by horizontal bars. $C$, Similar effects are shown for a different slice. The protonophore FCCP $(1 \mu \mathrm{M})$ appears to produce a more rapid mitochondrial depolarization and an average fluorescence increase that exceeded the initial level. The actual width of panels in $A$ is $3.3 \mathrm{~mm}$. The gray scale ( $A$, top right) ranges from 0 to 255 arbitrary fluorescence units (F1.U.). Similar results were obtained in eight other experiments.

DG (top middle), roughly along the perforant pathway. Figure $3, D$ and $E$, shows the SD plots corresponding to the mean data displayed in Figure $3, A$ and $B$, respectively, and reports changes in any direction (increase or decrease in fluorescence) that occurred in the stacks of images. The inset above Figure $3 D$ shows the electrical record over the sixth image collected for this portion of the analysis and shows sparse activity. There is little variation in the prestimulation series of images. The mean SD over this image was 0.0016 Fl.U. (range, 0-0.0059). The insets over Figure $3 E$ illustrate the stimulus train $(S)$ that was followed after a short delay by burst activity and a detail of the final burst. The variability in fluorescence across the six images collected after stimulation matches the pattern seen in the digital subtraction of images in Figure $3, A$ and $B$ (compare 

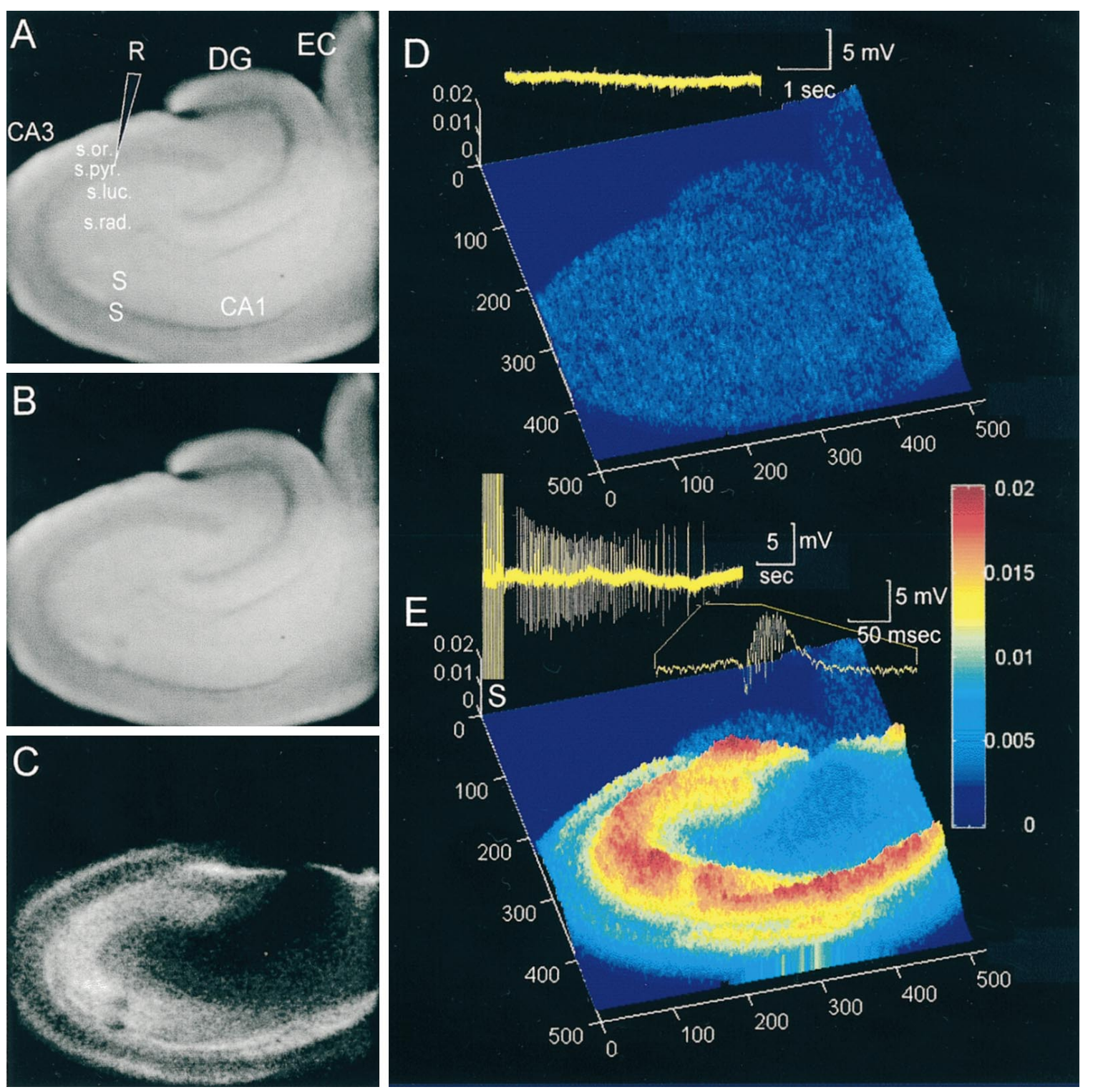

Figure 3. Electrical stimulation increases R123 fluorescence in the hippocampal slice in aCSF containing $0.9 \mathrm{~mm} \mathrm{Mg}^{2+}$. A slice was loaded with R123, and fluorescence changes were analyzed by obtaining periodic sets (stacks) of 30 images (128 video frame averages; $\sim 4$ sec each) as described in Materials and Methods. $A$ shows the digital average of the first six images, an image stack for baseline staining pattern. Note that the staining is fairly uniform and that the soma layers appear dark. Indicated are the stimulation electrodes $(S)$, a diagram of the recording electrode $(R)$, and the approximate locations of the $E C, D G$, s.or., s.pyr., s.luc., and s.rad. The width of the image is $3.3 \mathrm{~mm}$. $B$ is the mean fluorescence averaged over six images collected immediately after those used in $A$ and during and after application of a stimulus train (see Materials and Methods). $C$ is the digital difference of $B-$ $A$ and has been contrast enhanced to reveal regions of fluorescence increase. Note that most of the fluorescence increase is confined to the s.pyr., s.luc., and s.rad., as well as to a bright band of increase from the $E C$ through the $D G$. The brightest regions represent an increase of 3 Fl.U. $D$ shows a three-dimensional mesh plot of the pixel-by-pixel SD for the six images averaged in $A$. SD is encoded by both height on the $z$-axis and color and depicts the location and magnitude of fluorescence changes. The inset ( yellow) above the plot is the record of electrical activity during the final image just before electrical stimulation. $E$ shows the SD for $B$. The insets show the electrical record with the stimulus train $(S)$, a brief delay, and STIB activity that occurred during collection of the six images. Detail of the final burst is shown on a faster time base. The color scale used in $D$ and $E$ corresponds to SDs ranging from 0 to $0.02 \mathrm{Fl}$.U. The actual width of panels $A-E$ is $3.3 \mathrm{~mm}$. Data are representative of seven similar experiments.

Fig. 3C,E). The mean SD was increased nearly fourfold (mean $=0.0060 ;$ range, $0-0.0203$ ) over prestimulation values in this example.

Changes in fluorescence intensity after electrical stimulation were also observed with TMRM and TMRE, rhodamine dyes with a more linear response than R123 to mitochondrial potential (Ehrenberg et al., 1988). TMRM and TMRE are less likely to bind to mitochondrial proteins and to quench, thus making them more "well-behaved" dyes. Both spontaneous and stimulation- evoked changes in fluorescence were evident in slices constantly perfused with TMRM (100 nM) and TMRE (1 $\mu \mathrm{M}$ preload) (data not shown). Both the increases and decreases in fluorescence in response to synaptic activity observed with R123 were also observed with both other dyes. Despite its shortcomings, R123 has been used previously to reliably indicate changes in mitochondrial potential in neurons (Duchen, 1992). The unquenching of R123 may have the added advantage of emphasizing dye release, amplifying potentially small events. Because slices could be more 
conveniently studied after a single loading with R123 without the need for dye in the perfusate, most studies used R123.

Because different stimuli have been shown to change the intrinsic optical properties of tissue, we needed to account for changes caused by activity-dependent cell swelling. These changes are especially prominent in glia (e.g., MacVicar and Hochman, 1991; Andrew and MacVicar, 1994) and can be rapid after electrical stimulation (Lipton, 1973). In four slices not loaded with dye, the intrinsic change in fluorescence properties was either undetectable or up to a 1 Fl.U. change in the average slice signal under similar detection conditions, compared with the 2-10 Fl.U. average change commonly measured in R123-loaded slices (here approximately a $5 \%$ change). The intrinsic optical signals from slices loaded with the potential-insensitive dye NAO were similar to those from slices with no dye present (Fig. 4). Comparison of these images and analyses with those of rhodamine or rhod-2 signals (see below) reveals that activitydependent, intrinsic changes in the optical properties of the slice do not explain the fluorescence changes observed with mitochondrial potential-sensitive dyes under our observation conditions.

We determined the nature of the spontaneous change in rhodamine fluorescence signal in several slice preparations viewed under higher magnification. The level of spontaneous activity was variable, but it was clear that the fluorescence changes occurred at both bulk-tissue and single-cell levels. An example of spontaneous activity in the dentate/hilar region is shown in Figure 5. Activity is seen as the progressive increase and decrease in fluorescence that appears to also travel across cellular processes and between adjacent cells. This is best seen in the time-lapse movie of these images available in the electronic version of this paper (http://www.jneurosci.org/supplemental/18/12/4570). The confined, progressive changes suggest that the fluorescence changes are attributable to dye redistributions within single cells. This type of activity was also detected in other brain regions (data not shown) and is perhaps the result of local, spontaneous electrical activity.

At low magnification, the size and extent of the change in the R123 signal was proportional to the level of electrical activity in the slice. Consistent with the hypothesis that the change in the R123 signal was attributable to changes in electrical activity within the slice, addition of TTX blocked the stimulation-induced rise in R123 fluorescence (Fig. 6A). The SD plots in Figure $6 A$ show that electrical stimulation produced a band of change in the R123 signal (Fig. 6 $A 1$ ) that was blocked by the addition of TTX (FIg. 6A2). Recovery followed washout of TTX (data not shown). In these two-dimensional plots of fluorescence change, black indicates regions of no change, and increased gray scale brightness represents increasing levels of variability. The TTX sensitivity shows that the fluorescence increase is mediated by synaptic activity in the slice rather than by a direct depolarization caused by the electric field. The release of dye that accounts for the change in R123 signal was because of mitochondrial depolarization, because a similar signal was observed immediately after application of the mitochondrial uncoupler FCCP (Fig. 6A3), even though the pattern of change with FCCP was more general. A general increase in fluorescence was also observed after application of glutamate receptor agonists (data not shown).

Because antagonists of NMDA receptors block the induction of STIB (Anderson et al., 1987), it seemed likely that the changes in mitochondrial transmembrane potential caused by spontaneous or evoked electrical activity were also mediated, at least in part, by $\mathrm{Ca}^{2+}$ influx through glutamate-gated channels (see below; e.g., Ankarcrona et al., 1995; Bindokas and Miller, 1995; Khodorov et
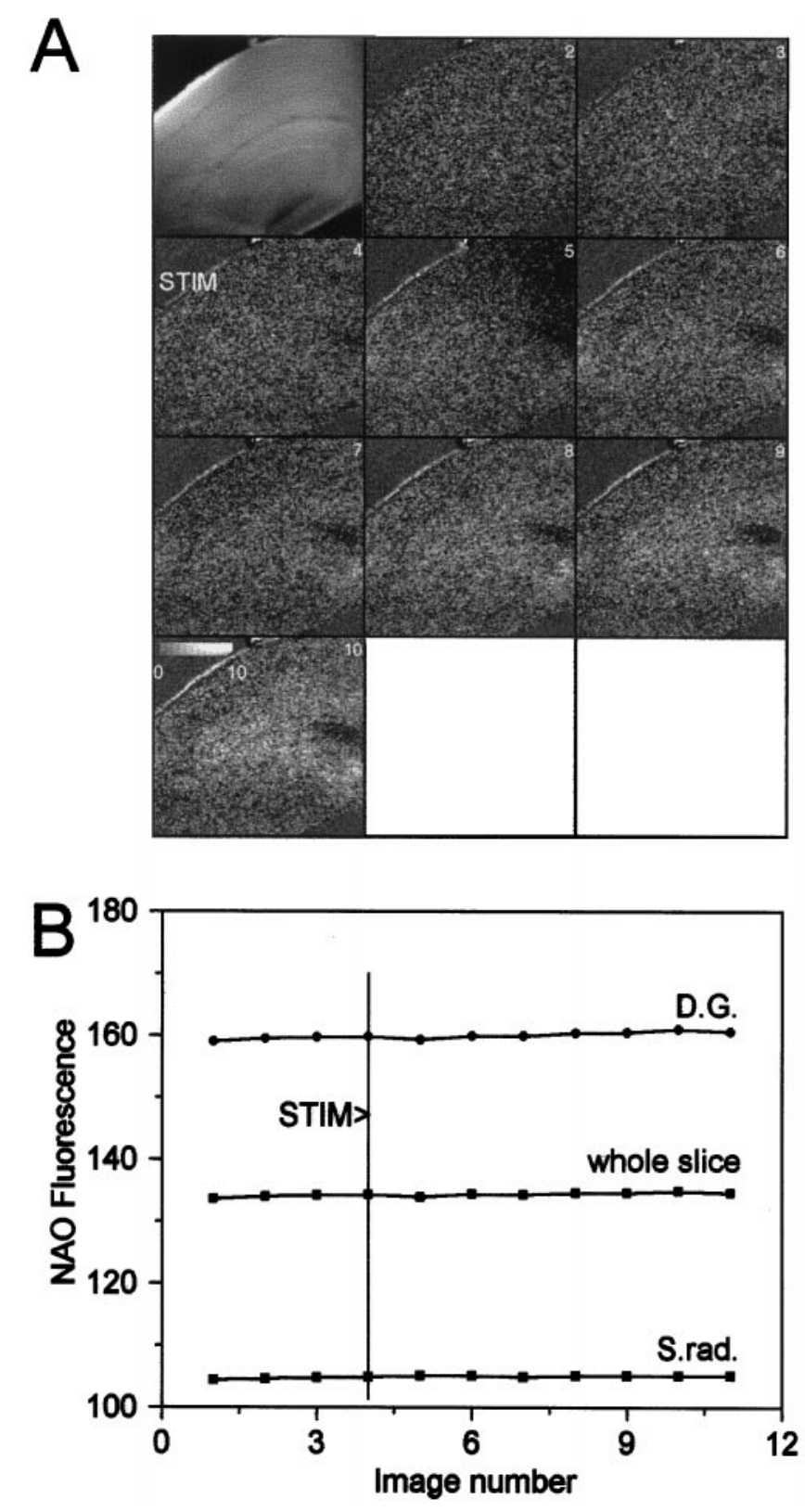

Figure 4. Changes in intrinsic optical properties produce relatively small changes in the fluorescence of potential-insensitive mitochondrial dyes. $A$, This slice was stained with the potential-independent, mitochondrialabeling stain NAO $(1 \mu \mathrm{M})$ and treated like the rhodamine-stained slices. The fluorescence image in the first panel shows a staining pattern similar to that seen with potential-sensitive mitochondrial dyes. $A 2-A 10$ show changes in fluorescence signal relative to this first image $\left(F_{n}-F_{1}\right)$. Electrical stimulation occurs during or within $A 4(S T I M)$. Note the small change in fluorescence with this potential-independent dye versus the relatively large changes in rhodamine signals (see Figs. 3, 6, 7 for a comparison) and in the rhod-2 signal (see Fig. 10). There are some small increases (lighter) and decreases in the fluorescence signal evident in $A 5-A 10$, but the mean change is of the same order of magnitude as that seen in unstained slices (i.e., the "intrinsic optical signal"; mean change = 1 Fl.U. in this example) and is much smaller than the rhodamine fluorescence signal we report. The image manipulations and the fluorescence scale bar ( $0-10 \mathrm{Fl}$.U.) shown in $A 10$ are identical to those used below (see Fig. 10). The original image width was $3.3 \mathrm{~mm}$. $B$, Quantitation of the NAO fluorescence changes is shown for the dentate gyrus (top), the average over the whole slice (middle), and for the s. radiatum near the stimulation electrode (bottom) for this example. Data are representative of three similar experiments. 

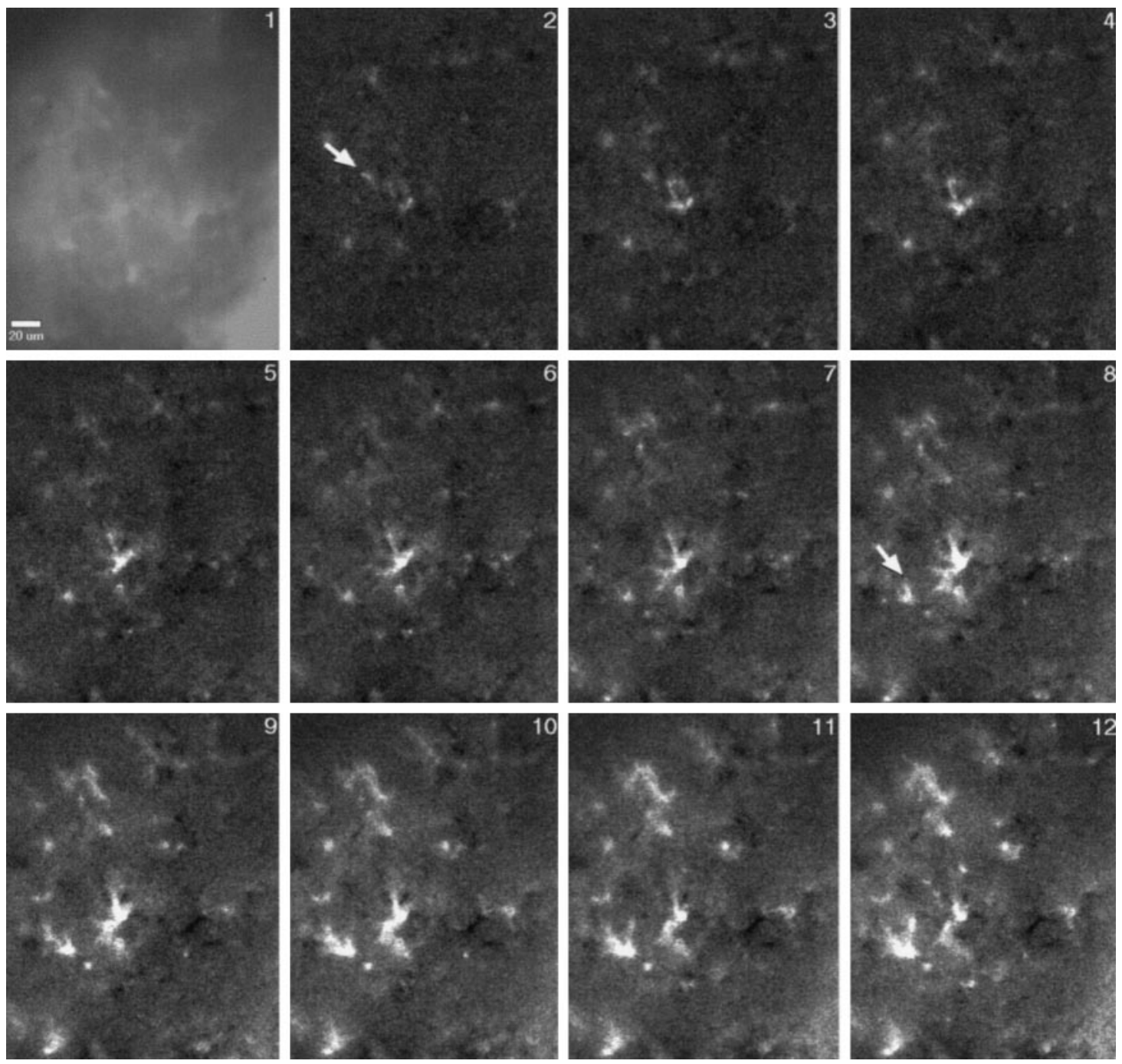

Figure 5. High-magnification $(40 \times)$ detail of the spontaneous changes in R123 fluorescence in a hippocampal slice. The type and degree of observed activity was highly variable from region to region and from preparation to preparation. Frame 1 shows the nonuniform staining pattern observed in the hilar region. The endal limb of the DG lies at the top and right edges of the image; CA3 lies just below the left bottom corner. This staining pattern was dynamic. Frames 2-12 show the relative change $\left(F_{n}-F_{1}\right)$ in fluorescence for 12 successive images (128 frame averages). Note that the fluorescence increases (brighter gray scale values) are limited to somata and their cellular processes, that they progress around single cells ( frames 2-7; cell indicated by arrow), and that they appear to propagate between cells ( frames $8-12$; arrow). Relative decreases in fluorescence (darker gray scale values) follow the increases. These details are best observed in digital movies available in the electronic edition of this paper (http://www.jneurosci.org/supplemental/18/ 12/4570). The data shown here span $70 \mathrm{sec}$ and represent raw fluorescence changes of up to 8 fluorescence units.

al., 1996; Schinder et al., 1996; White and Reynolds, 1996). The electrical and R123 responses were both low in aCSF containing $2 \mathrm{Mg}^{2+}$, both high in aCSF containing $0.9 \mathrm{Mg}^{2+}$, and both highest with the occurrence of ictal-like electrical discharges (see Fig. 11). Both responses reversed after wash in aCSF containing $2 \mathrm{Mg}^{2+}$ (data not shown). STIB in $0.9 \mathrm{mM} \mathrm{Mg}^{2+}$ commonly produced a broad, spreading increase in fluorescence and was sometimes accompanied by a propagating wave in s. oriens (see below). This $\mathrm{Mg}^{2+}$ sensitivity of the slice electrical activity was most likely caused by changes in glutamate-gated currents (Mayer and Westbrook, 1987) and electrical activity within hippocampal slices (Anderson et al., 1986; Walther et al., 1986). Application of D-AP-5 plus either CNQX or DNQX blocked the stimulationevoked rise in R123 fluorescence. Figure $6 B$ shows mean derivative plots in which an increased gray scale brightness indicates an increase in R123 fluorescence, middle gray indicates no change, and dark gray values indicate regions of net decrease in fluores- cence. Figure $6 B 1$ shows that there was a net mitochondrial depolarization, consonant with $\mathrm{Ca}^{2+}$ influx, along the s pyr. after stimulation and a band of fluorescence decrease in the s.oriens of CA3 and in DG. The application of glutamate antagonists eliminated STIB and the fluorescence increase along the s.pyr. The dark bands may represent a relative "repolarization" of mitochondria, perhaps recovering from spontaneous electrical events occurring just before data collection.

A subset of slices $(n=26)$ were observed to show spontaneous waves of mitochondrial depolarization in both 2 and $0.9 \mathrm{~mm}$ $\mathrm{Mg}^{2+}$ aCSF. These occurred most often in slices with apparently intact tracts from the EC to the DG and were less common in slices with no EC. Most commonly, these waves originated in the EC and progressed through the DG following the mossy fiber tract within the s.luc. into the s.rad. of the CA3 and ultimately the CA1 regions. The spontaneous waves could dominate the response of a slice compared with stimulation-induced rises, and 

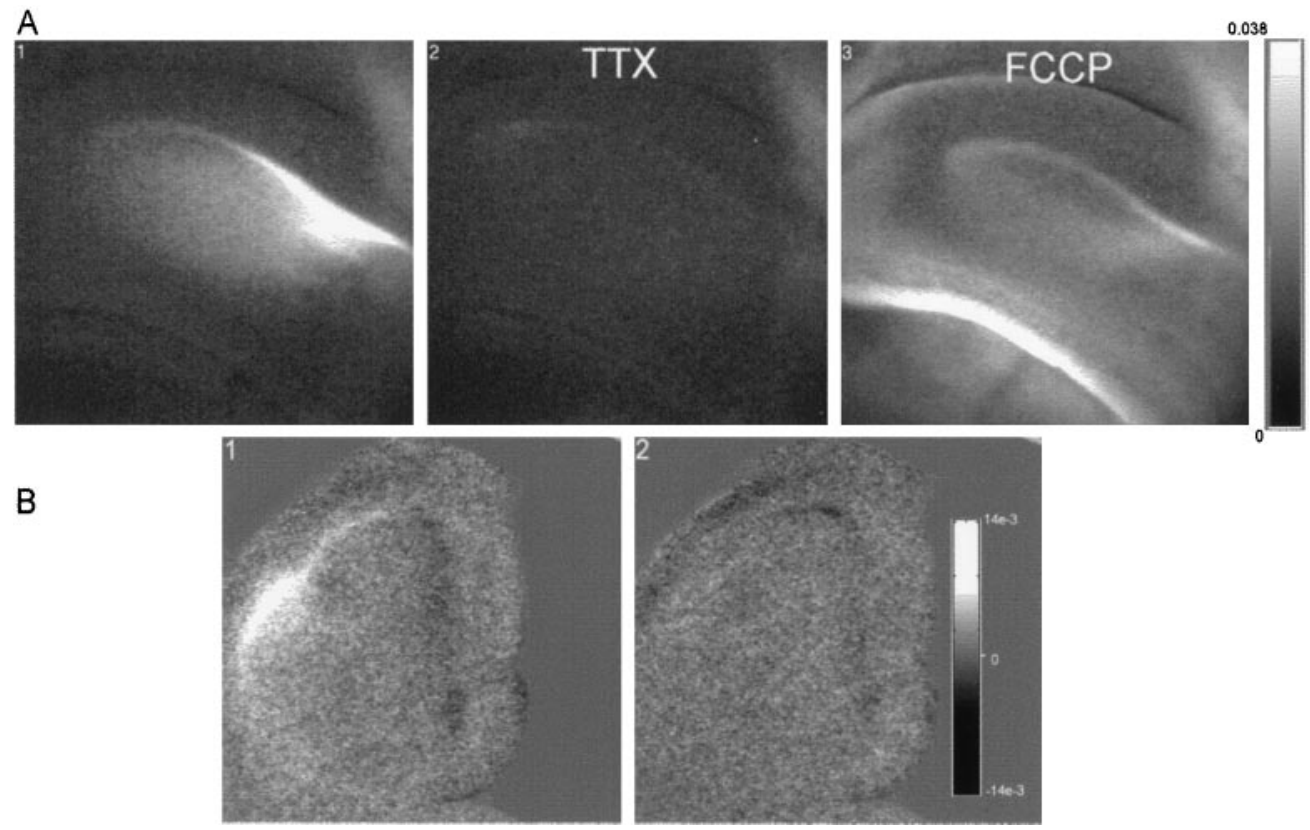

Figure 6. Stimulus-associated increase in R123 fluorescence is proportional to electrical activity. $A$ shows average SD plots (see Materials and Methods) for fluorescence across an image stack after electrical stimulation and after application of $T T X$ or $F C C P$. A1 shows the basal response in which fluorescence increased (data not shown) after stimulation. Mean SD was 0.0069 Fl.U. (range, 0-0.0387), and most of the change was limited to a band near CA1 (top). A2 shows that TTX (1 $\mu \mathrm{M})$ added for $5 \mathrm{~min}$ prevented the localized changes in fluorescence after electrical stimulation. STIB activity was suppressed (data not shown). Mean SD was 0.0045 (range, $0-0.0141$ ). A3 shows that after washout of $T T X$ and recovery (data not shown), application of the mitochondrial uncoupler FCCP $(1 \mu \mathrm{M})$ immediately produced more general increases over the entire slice and especially along the lower soma layer. Mean SD was 0.011 (range, 0-0.0377). Black represents no change (0), and white is SD $\geq 0.038$. Data are representative of three similar experiments. $B$ shows mean derivative plots before $(B 1)$ and 10 min after $(B 2)$ treatment with D-AP-5 $(50 \mu \mathrm{M})$ plus CNQX (10 $\mu \mathrm{M})$. The control plot (B1) shows a net increase in fluorescence in the CA1 and CA2/3 region (bright band) and a fluorescence decrease (dark band) from DG to CA3b after stimulation that resulted in STIB (26 bursts; data not shown). Glutamate antagonists eliminated the STIB activity after stimulation (0 bursts) and also the increase in fluorescence. The grand average of all mean derivatives was $1.4 \mathrm{e}^{-4}$ (range, $-4.1 \mathrm{e}^{-4}$ to $1.4 \mathrm{e}^{-3}$ ) before and $7.9 \mathrm{e}^{-5}$ (range, $-5.4 \mathrm{e}^{-4}$ to $6.8 \mathrm{e}^{-4}$ ) after glutamate antagonists in this example. The actual width of frames is $3.3 \mathrm{~mm}$. Data are representative of five similar experiments.

their presence before a STIB induction attempt would preclude STIB activation. Spontaneous electrical activity resembling interictal discharges sometimes accompanied these R123 waves and was more prevalent in $0.9 \mathrm{~mm} \mathrm{Mg}^{2+}$ aCSF. A previous study of this preparation has shown that interictal activity can suppress the generation of seizures (Bragdon et al., 1992). Spontaneous waves of mitochondrial depolarization within thick hippocampal slices thus seem to be caused by endogenous electrical activity.

The pattern and progression of both the spontaneous and the stimulation-evoked changes in R123 fluorescence were similar. Because the changes occurred repeatedly, it is likely that the changes in mitochondrial potential were short-lived to allow recapture of most of the R123 released by depolarization before dye washout from the slice. However, this recapture was apparently incomplete because there was a slow loss of signal from the slice over the course of long experiments. This loss was relatively uniform over the slice (see below; see Fig. 12, top), suggesting that activity-dependent loss of dye was a minor constraint on repeated measurements. It is also likely that the mitochondrial depolarizations were not complete, so that either the same population of mitochondria produced incremental dye release or different mitochondrial populations within the same cells were depolarized during different events. Spontaneous, and some stimulationevoked, waves were long lasting and were easily detected with the slow sampling rates used in most experiments. Spontaneous waves moved at an average velocity of $29 \pm 2 \mu \mathrm{m} / \mathrm{sec}$ (measured in three slices) when moving along the pyramidal layers in CA1 and CA3. Lateral spread of some waves, as in Figure $7 A$, was slower $(5 \pm 0.5 \mu \mathrm{m} / \mathrm{sec} ; n=13)$. The propagation of the waves was often saltatory, seeming to linger in certain regions (like area $\mathrm{CA} 3 \mathrm{c}$ ) and then progressing rapidly through other regions. Consequently, wave velocity distributions were skewed toward faster velocities, but the true extent of the skew could not be accurately measured with the slow imaging frequency. Stimulationassociated waves near the electrode were likewise too fast for accurate measurement. Furthermore, wave fronts were difficult to distinguish in images made from shorter-duration averages.

Comparison of the stimulated and spontaneous activity in a representative slice is shown in Figure 7 in which the images in $A$ and $C$ show the response to stimulation and in $B$ and $D$ show spontaneous changes occurring $5 \mathrm{~min}$ after the data in $A$ and $C$ were obtained. Changes in mitochondrial potential could last the full 2 min of data collection, as can be seen by the prolonged increase in R123 fluorescence in Figure $7 A$. The dark band in the DG visible in frame 2 (Fig. $7 A$ ) is the result of loss of fluorescence of a spontaneous wave that was waning in intensity and that had occurred just before acquiring this data set. Thus, the first image was brighter than images $2-30$ in that region. Application of a stimulus train to the $\mathrm{CA} 2 / 3$ region (Fig. $7 A$, frame 6 , near the bottom left) produced a small increase in fluorescence (frame 7) in the immediate vicinity of the electrodes. Note that a spontaneous increase in fluorescence had begun in that vicinity just before stimulation. This wave of mitochondrial depolarization spread toward the CA1 region and more strongly to CA3. The wave rapidly progressed toward the DG where it lingered and spread laterally in CA3, whereas it deceased in intensity near the 


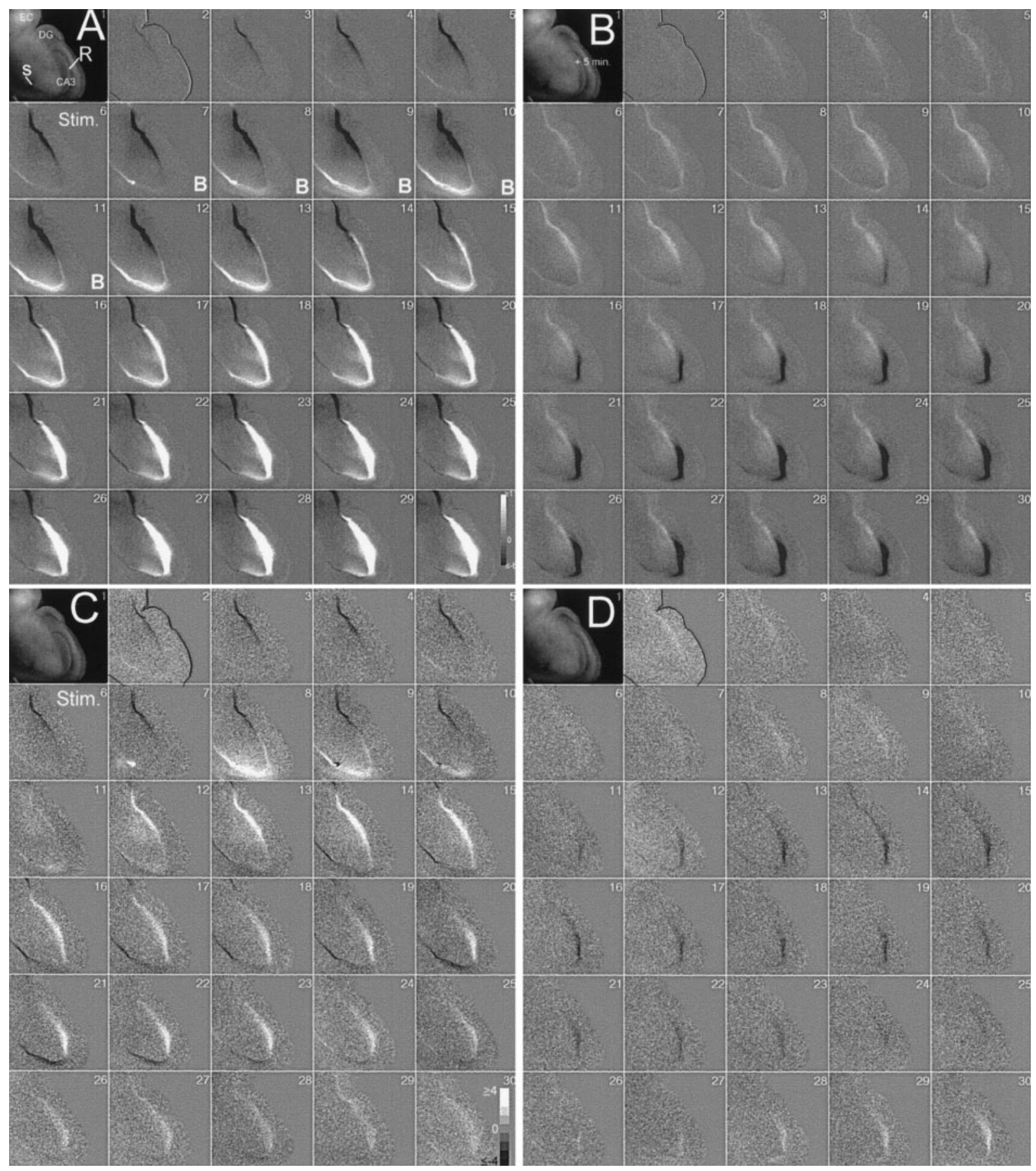

Figure 7. Stimulation produces larger and longer-lasting changes in mitochondrial activity compared with spontaneous electrical activity. Four sets of 30 images are shown. $A, B$, The incremental change in R123 fluorescence compared with the initial staining fluorescence $\left(F_{n}-F_{1}\right)$ shown in frame 1 of each series. These image sets reveal net fluorescence changes compared with the initial image over the entire 2.5 min data collection period. $C, D$, The reanalyses of the same data sets shown in $A$ and $B$ showing the fluorescence change between "successive" images $\left[F_{n}-F_{(n-1)}\right]$. These lower sets show fluorescence changes over $\sim 8 \mathrm{sec}$ intervals. Data from the stimulated $(A, C)$ and nonstimulated $(B, D)$ preparation $(5$ min after the collection of $A, C$, respectively) are shown. The slice outline is visible and constant within these digitally subtracted images. The background is uniform and constant, whereas the area within the slice is filled with fine-grained noise producing the "salt-and-pepper" fill pattern. Slice edges are also manually outlined in the second frame within each data set. Stimulation $(A 6)$ produced STIB activity during $A 7-A 11$ (record not shown; frames labeled $B$ ) and a more intense and longer-lasting increase in fluorescence compared with that in the nonstimulated data set (image set $B$ ). Reanalysis $(C)$ of the data in $A$, displaying the differences between successive images, reveals that the apparently constant changes are because of a series of faster, wave-like mitochondrial depolarizations masked by a persistent depolarization. The stimulated rise subsides as the STIB ended during C11, and a new wave invaded from the EC toward the CA3. This wave waned for $C 16-C 20$, briefly revived $(C 21-C 24)$, and then finally stopped despite the persistent depolarization still present in CA3 (see $A 30$ ). For spontaneous activity shown in $B$, this analysis method revealed a depolarization wave in $D 26-D 30$, a period when the net fluorescence was lower than the initial level (see B26-B30). Scale bars for gray scale coding are shown in $A 30$ and $C 30$ for actual (raw) fluorescence changes occurring in $A$ and $B$ and in $C$ and $D$, respectively, and are constant for each type of data presentation $(A=B ; C=D)$. The actual width of frames is $3.3 \mathrm{~mm}$. Data are representative of 16 similar experiments. Data are available as time-lapse movies in the electronic edition of this paper (http://www.jneurosci.org/supplemental/18/12/4570). 
site of origin in CA2/3. Spontaneous activity ( $5 \mathrm{~min}$ later) is shown in Figure $7 B$. The level of activity is lower, but the path and pattern of spread are nearly identical to the stimulus-evoked response.

Reanalysis of this data by means of sequential pair differences revealed changes occurring over $\sim 9$ sec intervals (Fig. $7 C, D$ ) versus the cumulative changes from the initial image (up to 2.5 min) shown previously in Figure 7, $A$ and $B$. In Figure $7 C$, the stimulus was seen to produce a wave that propagated along CA1 toward the subiculum and also along CA3 toward the DG. This was followed by a wave that invaded from the EC through the DG and into area CA3. This second wave appeared to oscillate before the final decrease. Similarly, a small spontaneous wave became evident with the finer time resolution in Figure $7 D$. The small wave now visible in the final five frames was obscured by the net decrease in fluorescence intensity seen in Figure $7 B$. Both types of analysis were thus required for the detection of long- and shorter-term changes in mitochondrial potential.

The mean fluorescence intensity plots for six slice regions from the images in Figure 7, $A$ and $B$, are presented in Figure 8. Figure $8 A$ shows raw data for the mean fluorescence in various regions of the slice corresponding to the analysis shown in Figure $7 A$. The transformation of this data to a derivative plot in Figure $8 B$ better reveals the size and sequence of the relatively small fluorescence changes in Figure $8 A$ that followed electrical stimulation. The data from cumulative change plots shown in Figure $7 A$ are plotted for the same slice regions and shown in Figure $8 C$. As in the images (Fig. $7 A$ ), it is clear from the intensity plots that the CA3 region had a prolonged change in mitochondrial potential (Fig. 8A,C). The depolarization began at the stimulation site (arrow) and progressed sequentially away toward DG. Plots from the pairwise-change images (Fig. $7 B$ ), shown in Figure $8 D$, yield data identical to the derivative plot obtained from the raw fluorescence data (compare Fig. $8 B, D$ ). The oscillation in mitochondrial potential toward the end of the record is clearly visible (arrow).

\section{Changes in $\left[\mathrm{Ca}^{2+}\right]_{\mathrm{i}}$}

Our implicit assumption is that mitochondria are depolarized as a consequence of electrical activity within the slice. Mitochondrial potential is dissipated by the influx of $\mathrm{Ca}^{2+}$ ions via the $\mathrm{Ca}^{2+}$ uniporter when in excess of $\mathrm{Ca}^{2+}$ efflux (Nicholls and $\AA$ Akerman, 1982). The duration of the mitochondrial depolarization observed here was also similar to the duration of elevations in $\left[\mathrm{Ca}^{2+}\right]_{\mathrm{i}}$ after tetanic stimulation of hippocampal slices (Miller et al., 1996). We hypothesized that an increase in $\left[\mathrm{Ca}^{2+}\right]_{\mathrm{i}}$ should occur in the same regions that exhibit mitochondrial depolarizations. This was tested in seven slices after loading with the modified rhodamine dye and fluorescent $\mathrm{Ca}^{2+}$ indicator rhod-2. Rhod-2 may be sequestered within energized mitochondria (e.g., Babcock et al., 1997) and may thus preferentially report mitochondrial $\left[\mathrm{Ca}^{2+}\right]\left(\left[\mathrm{Ca}^{2+}\right]_{\mathrm{m}}\right)$. Indeed, rhod-2 staining was nonuniform in slices and resembled that of potentiometric rhodamine dyes (Fig. 9). The distribution of rhod-2 exactly matched that of mitochondrial dyes with intense staining of the s.luc. and low staining of s.pyr. (compare Figs. $1 B$, 9). Experiments in cultured hippocampal pyramidal neurons, however, revealed that rhod-2 (like other rhodamine dyes) appears to be released from mitochondria when they are depolarized (data not shown). Thus, rhod-2 signals can possibly arise from more than one cellular compartment.
An example of one slice experiment using rhod-2 conducted in $0.9 \mathrm{Mg}^{2+} \mathrm{aCSF}$ is shown in Figure 10. Stimulation occurred during the acquisition of frame 6 , and an increase in $\left[\mathrm{Ca}^{2+}\right]_{\mathrm{i}}$ was first evident in frame 7 near the stimulation electrode (the brightest part visible). Note that the stimulus-induced rise was shortlived and had primarily abated by frame 9 . A new increase began in frame 10, and STIB activity was recorded during frames 12-19 (electrical record not shown). This produced a wave of fluorescence increase that progressed in the pyramidal layers of CA3c and in the s.oriens toward CA3b. This is best seen in the timelapse movie of the images available in the electronic version of this paper (http://www.jneurosci.org/supplemental/18/12/4570). We did not determine the exact subcellular compartment(s) from which these relatively macroscopic signals arose. Nevertheless, the late, STIB-associated increase was long-lived and greatest in the s.rad. and s. lacunosum/moleculare and thus similar to the location of the major mitochondrial depolarization. These data support the hypothesis that the mitochondrial depolarizations observed with rhodamine dyes were attributable to corresponding $\left[\mathrm{Ca}^{2+}\right]_{\mathrm{i}}$ elevations.

\section{PYY blocks STIB and associated changes in mitochondrial potential}

NPY has recently been shown to suppress seizure activity in this type of slice preparation (Klapstein and Colmers, 1997) and in vivo (Woldbye et al., 1996, 1997), and NPY knock-out mice are prone to seizures (Erickson et al., 1996). Presynaptic NPY receptors suppress excitatory transmission in the hippocampus (Colmers et al., 1987; Bleakman et al., 1992; Qian et al., 1997). We therefore investigated effects of the potent NPY receptor agonist PYY on R123 activity. Figure 11 shows that the magnitude of the R123 response was proportional to the electrical activity recorded in the $\mathrm{CA} 3 \mathrm{c}$ region. The figure shows the electrical record (red trace) above each three-dimensional, colorized, SD plot. Although the electrode is sensitive to electrical activity only in its immediate vicinity, imaging was able to detect changes over the entire field of view. Thus, the enhancement of R123 fluorescence increase near the stimulation electrode that occurred before actual STIB recording in CA3 in $0.9 \mathrm{~mm} \mathrm{Mg} \mathrm{Mg}^{2+}$ aCSF (top right) probably reported an increase in local electrical activity that had not yet propagated to the recording site. Note that the size and extent of the R123 signal increased with increases in STIB intensity and duration. Application of PYY decreased both the electrical activity and the size of the R123 signal. In this example, the inhibitory effect on both the electrical and R123 effects diminished in the continued presence of PYY (possibly because of the imperfect perfusion of the slice in the inverted chamber) (cf. Klapstein and Colmers, 1997). After washout of the peptide, the electrical and R123 signals recovered and exceeded pre-PYY levels. This may have been because of continued slow washout of $\mathrm{Mg}^{2+}$ from the slice or perhaps because of kindling.

The full time course for the experiment shown in Figure 11 is shown in Figure 12. Figure 12 (top) shows the fluorescence intensity for regions of the CA3b,c s.oriens (diamonds, top trace), the CA1c s.oriens (triangles, bottom trace), and CA3a s.luc. at the stimulation electrode site (squares, middle trace). Note that two stimulations in $2 \mathrm{Mg}^{2+}$ aCSF resulted primarily in rises only near the electrode (middle trace) and that the R123 signal slowly declines during the $>3 \mathrm{hr}$ experiment, presumably from dye washout and/or bleaching. Reduction of $\left[\mathrm{Mg}^{2+}\right]_{\mathrm{o}}$ resulted in an 

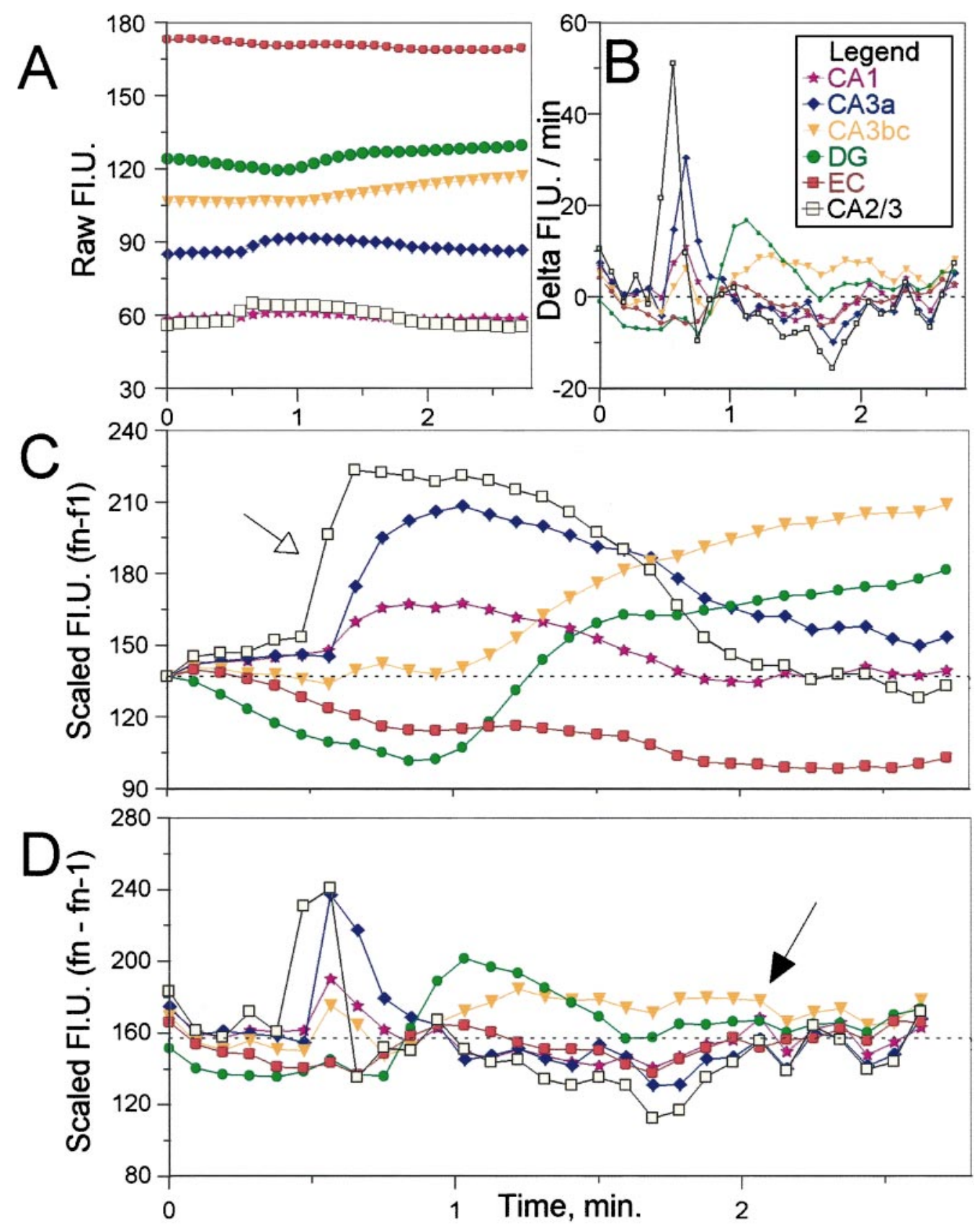

Figure 8. Raw data intensity plots and analyses of data presented in Figure 7, $A$ and $B . A, B$, Raw mean intensities for regions of the slice $(A)$ and the corresponding first derivative plots for this raw data $(B)$. The legend is common to all panels. Electrical stimulation produced a large, fast depolarization first in the CA2/3 region (squares) near the electrode and slower, delayed rises in other regions $(B)$. $C$, The corresponding cumulative changes in potential for scaled data from Figure $7 A$. This plot likewise identifies regions of increased and decreased R123 fluorescence relative to the initial image in the data set. The rise near the stimulation site was most rapid and long-lasting (arrow). The dotted reference line is the gray level indicating no change. $D$, The corresponding plots from the successive, pairwise difference images (Fig. 7B). The data are essentially the same as the derivative plot from raw data (compare with $B$ ). Note the oscillation in mitochondrial potential during the final $1 \mathrm{~min}$ of the plot (e.g., arrow). The dotted reference line is the gray level indicating no change.

increasing stimulation-associated rise in the s.oriens, especially in the CA1 (bottom trace). PYY decreased the rise at all three sites, but mostly at the s.oriens (arrow). It also altered the kinetics of the R123 changes as shown in greater detail for the CA1 s.oriens trace (Fig. 12, bottom). PYY delayed the onset of the rise by perhaps $10 \mathrm{sec}$, decreased the amplitude of the rise, and slowed the recovery rate. Overall, PYY reduced electrical and R123 responses in seven out of seven slices.

\section{DISCUSSION}

We have demonstrated that brief electrical events (especially epileptiform discharges) produce relatively long-lasting changes in rhodamine fluorescence (mitochondrial potential), as well as associated increases in $\left[\mathrm{Ca}^{2+}\right]_{i}$ in the hippocampal slice. Mitochondrial potentiometric dyes provide a useful new tool for mapping patterns and time courses of synaptic activation in a 


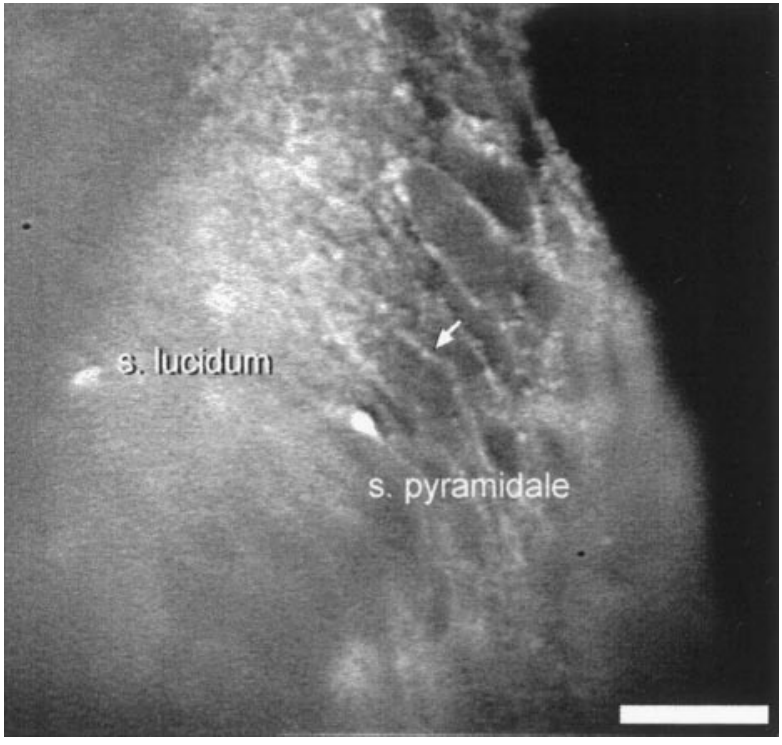

Figure 9. Distribution of fluorescence signal from a hippocampal slice loaded with rhod-2 AM is identical to that obtained with potentiometric rhodamine dyes. This high-magnification image of an obliquely sectioned slice shows sparse staining of the large CA3 pyramidal neuronal somata, punctate staining at the margins of the somata (arrow), and more intense staining of the s.luc. region. Compare this staining pattern with that of $\mathrm{R} 123$ shown in Figure $1 B$. Scale bar, $50 \mu \mathrm{m}$.

variety of neuronal preparations, as well as the functional consequences of synaptic activity.

R123, a probe that stains mitochondria in a Nernstian manner (Emaus et al., 1986; Duchen and Biscoe, 1992), reported changes in mitochondrial transmembrane potential within slices. Collapse of mitochondrial potential with protonophores like FCCP releases dye and initially increases fluorescence (Duchen, 1992). However, prolonged depolarization leads to loss of dye from the cell (e.g., Duchen and Biscoe, 1992). During normal cellular activity, the electrogenic, uniporter-mediated uptake of $\mathrm{Ca}^{2+}$ by mitochondria decreases their transmembrane potential (Nicholls and Åkerman, 1982). The increased $\left[\mathrm{Ca}^{2+}\right]_{\mathrm{m}}$ stimulates activity of the tricarboxylic acid cycle, and the resulting recovery of mitochondrial potential seems to enable recapture of R123 during phasic changes in $\left[\mathrm{Ca}^{2+}\right]_{\mathrm{i}}$. Although this dye may not be ideal for the calculation of mitochondrial potential due to its binding and partial quenching, the unquenching that occurs after dye release is useful for emphasizing mitochondrial depolarizations. We obtained similar optical records with TMRM and TMRE (data not shown), dyes with less binding and quenching (Ehrenberg et al., 1988).

We have shown previously that glutamate application depolarized mitochondria as a result of $\mathrm{Ca}^{2+}$ accumulation within neurons in culture (Bindokas and Miller, 1995). In the present experiments, agonists at glutamate-gated channels and FCCP both increased R123 fluorescence. Synaptically activated increases in $\left[\mathrm{Ca}^{2+}\right]_{\mathrm{i}}$ and/or $\left[\mathrm{Ca}^{2+}\right]_{\mathrm{m}}$ measured with rhod- 2 occurred at similar locations and with similar durations as the changes in mitochondrial potential reported by R123. Synaptically mediated mitochondrial depolarization in the brain probably arises from mitochondrial uptake of $\mathrm{Ca}^{2+}$ as shown in other preparations (Loew et al., 1994; Bindokas and Miller, 1995; White and Reynolds, 1996). Moreover, rhod-2, the dye used to image $\left[\mathrm{Ca}^{2+}\right]_{\mathrm{i}}$ in present studies, may preferentially accumulate within mitochon- dria (e.g., Babcock et al., 1997) and may thus predominantly report $\left[\mathrm{Ca}^{2+}\right]_{\mathrm{m}}$. High-magnification images confirmed a nonuniform distribution of dye in slices, with little dye in the mitochondria-sparse somata of pyramidal neurons (Fig. 9). It is therefore not surprising that patterns of mitochondrial depolarization and $\left[\mathrm{Ca}^{2+}\right]_{\mathrm{i}}$ increases were similar. Blockade of synaptic activity with TTX, glutamate antagonists, or PYY reduced both the electrical activity and the mitochondrial depolarization. Because mitochondrial depolarizations can also arise from metabotropic $\mathrm{Ca}^{2+}$ release in addition to $\mathrm{Ca}^{2+}$ influx (e.g., Rizzuto et al., 1993), it is possible that some of the observed mitochondrial depolarizations arose from $\mathrm{Ca}^{2+}$ released from intracellular stores (e.g., MacVicar and Tse, 1989; Jaffe and Brown, 1994; Taylor et al., 1995; Miller et al., 1996).

Burst discharges produced waves of mitochondrial depolarization within slices that far outlasted the electrical events. Electrical stimulation producing epileptiform discharges resulted in depolarization of mitochondrial potential in cells limited to layers known to be important in the generation and maintenance of the burst discharges (Bragdon et al., 1992). The mitochondrial activity was greatest in s.rad., and the s.oriens component was markedly increased, whereas the s.pyr. showed less depolarization. The pattern of mitochondrial depolarization was similar to that of fast cellular depolarizations recorded by voltage-sensitive dyes after 4-aminopyridine administration (Barish et al., 1996). Those dye responses are typically small and too rapid to reliably capture with conventional video microfluorimetry but can be resolved with photodiode arrays (e.g., Orbach and Cohen, 1983; Barish et al., 1996; Iijima et al., 1996; Senseman, 1996). In contrast, the mitochondrial consequences of these events were readily detected. Whereas the time course of the electrical events during a paroxysmal burst was completed within seconds, the mitochondrial depolarizations we observed routinely persisted for tens of seconds and could last minutes. Wave-like progressions of mitochondrial depolarization and repolarization followed appropriate anatomical tracts at rates $(5-40 \mu \mathrm{m} / \mathrm{sec})$ that were more similar to the $\mathrm{Ca}^{2+}$ diffusion rate [cf. Jaffe (1993); range, $\sim 15 \mu \mathrm{m} / \mathrm{sec}$ (Dani et al., 1992; Gabso et al., 1997) to 100-200 $\mu \mathrm{m} / \mathrm{sec}$ (Charles et al., 1996)] than the causative electrical events $[\sim 9 \mathrm{~cm} / \mathrm{sec}$ (Chervin et al., 1988; Iijima et al., 1996)]. Mitochondrial waves were associated with both stimulated and spontaneous electrical activity.

High-magnification studies suggest that R123 signals can be confined to cells with neuronal appearance (Fig. 5). Although the $\mathrm{R} 123$ signal in slices is probably not limited to neurons, the extent of the glial contribution to observed events is presently unclear. Glial cells abutting cultured neurons exhibit robust R123 fluorescence oscillations (V. P. Bindokas, unpublished observations). $\mathrm{Ca}^{2+}$ waves have been described in glia in hippocampal slices (Dani et al., 1992; Porter and McCarthy, 1996); thus a glial contribution to the macroscopic signals we observed is certainly possible. It is noteworthy that AM ester loading of slices with $\mathrm{Ca}^{2+}$ indicators appears to preferentially load astrocytes (e.g., Porter and McCarthy, 1996). This signal was not exclusive to glia in present studies, as judged by the apparent staining of mossy fiber and other synaptic terminals (Fig. 9; Bindokas, unpublished observations). Regardless, although the glial contribution to the $\left[\mathrm{Ca}^{2+}\right]$ reported by rhod-2 in the slice may be significant, both result from neuronal activity. Because the mitochondrial potential waves had a similar pattern to the $\mathrm{Ca}^{2+}$ waves, it likewise implies that glia and neurons may be responding in concert. Given the close trophic/homeostatic dependence of neurons with 

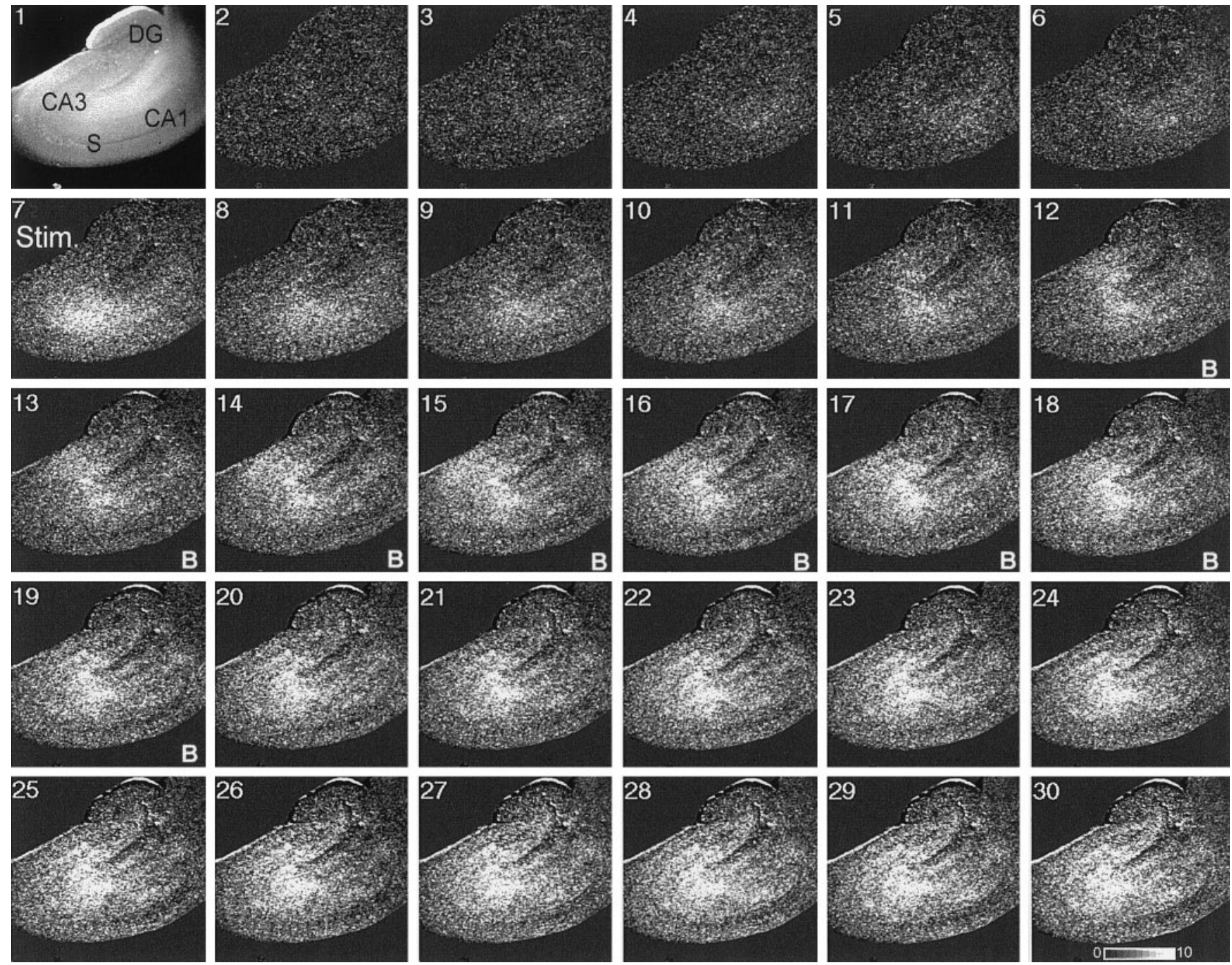

Figure 10. Changes in $\left[\mathrm{Ca}^{2+}\right]_{\mathrm{i}}$, measured with rhod-2 (see Materials and Methods), occur with spatial and temporal patterns similar to that of the mitochondrial depolarizations. Images show the relative fluorescence changes compared with the first image. Electrical stimulation (frame 7; STIM) produced a delayed STIB response during frames $12-19(B)$. Stimulation electrodes were situated as indicated in frame $1(S)$. The increase in $\left[\mathrm{Ca}^{2+}\right]_{\mathrm{i}}$ decreased after stimulation ( frames 8-10) and increased again just before recording STIB activity ( frame 10). The average increase over the entire slice was 2.1 Fl.U. and was 4.5 Fl.U. within the s.rad. Gray scale for the composite montage was optimized for image contrast (scale bar range, 0 to $\geq 10$ Fl.U.). Data are representative of three similar experiments. The actual width of panels is $3.3 \mathrm{~mm}$. Data are available as time-lapse movies in the electronic edition of this paper (http://www.jneurosci.org/supplemental/18/12/4570).

supportive glia (Westergaard et al., 1994, 1995; Magistretti and Pellerin, 1996; Tsacopoulos and Magistretti, 1996), changes in mitochondrial state in both cell types may be equally important for maintenance of brain activity (e.g., Parpura et al., 1994; Hassinger et al., 1995).

Does a reciprocal relationship between mitochondrial activity and synaptic activity play a critical role in the CNS? Mitochondrial energy production is homeostatically linked to synaptic activity. In addition, one can envision a role for mitochondria in synaptic plasticity (Miller, 1991) because these organelles are ideally situated to integrate responses to, and perhaps influence, both pre- and postsynaptic events. In support for a role of mitochondria in the control of synaptic events, mitochondrial involvement in synaptic fatigue and post-tetanic potentiation has been reported (Nguygen and Atwood, 1994; Tang and Zucker, 1997).

In the present study, the changes in mitochondrial state result- ing from synaptic activity observed may represent a previously unrecognized level of signaling or signal integration that is available to both pre- and postsynaptic neurons and supporting glia. Because mitochondria are tuned to phasic changes in $\left[\mathrm{Ca}^{2+}\right]_{i}$ such as those associated with action potentials (Sparagna et al., 1994; Hajnóczky et al., 1995) and because the resulting alterations in mitochondrial potential/functional state outlast the causative events, the mitochondrial changes reflect the integral of recent synaptic activity and changes in cytosolic $\left[\mathrm{Ca}^{2+}\right]$. A rapid release of accumulated $\mathrm{Ca}^{2+}$ via the mitochondrial permeability transition pore may contribute to $\mathrm{Ca}^{2+}$ signaling (cf. Bernardi and Petronilli, 1996; Simpson and Russell, 1996; Ichas et al., 1997). The ability of mitochondria to act as electrically excitable organelles capable of generating waves of traveling depolarization and $\mathrm{Ca}^{2+}$ release has recently been shown in vitro (Ichas et al., 1997). The sensitivity of mitochondria for phasic, high elevations 

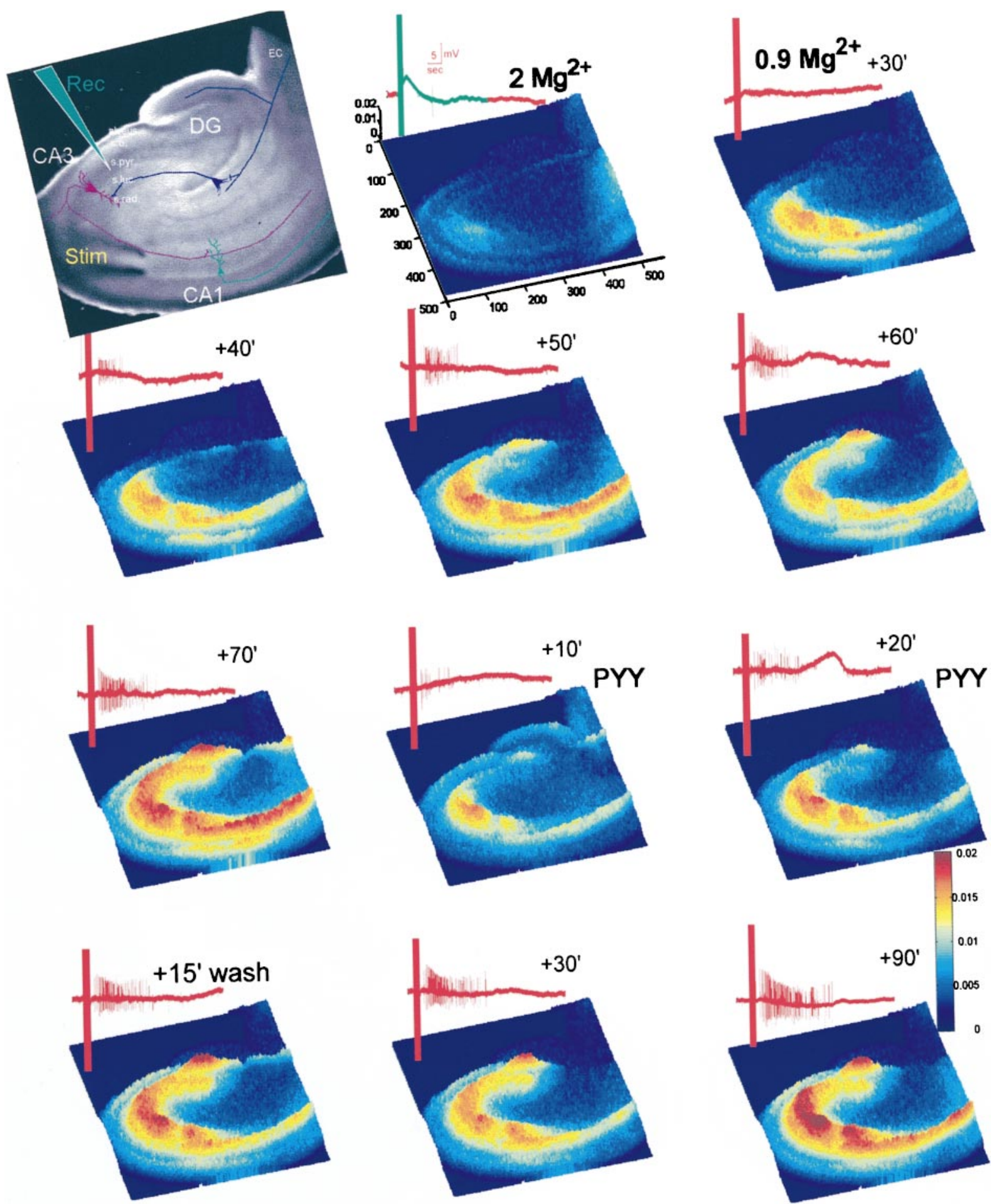

Figure 11. Mitochondrial depolarization is proportional to electrical activity and sensitive to effects of the neuropeptide PYY. The slice was loaded with R123 and stimulated via electrodes (visible in the top left, Stim). Illustrated are the major connections between regions in the slice. Electrical recordings were obtained from a micropipet situated in the s.pyr., as indicated (Rec), and are shown as the red insets above each panel. Each panel is a SD plot of the six images collected during and after the electrical stimulation. The plots show the location and magnitude of changes in mitochondrial potential. Stimulation in aCSF with $2 \mathrm{~mm} \mathrm{Mg}{ }^{2+}$ (mid upper row) produced a small mitochondrial depolarization in the vicinity of the electrodes. The region of the electrical record highlighted in green indicates the approximate time span over which the plotted data were collected in all panels. The $z$-axis of each panel corresponds to a maximum SD of 0.02 Fl.U. The third panel (top right) shows increased mitochondrial depolarization after electrical stimulation 30 min after changing to aCSF containing $0.9 \mathrm{~mm} \mathrm{Mg}{ }^{2+}$. Panels 4-7 (second row and third row, left) were recorded at 10 min intervals and show progressively greater regenerative electrical activity elicited by the stimulus train, concomitant with an increase in the extent and magnitude of mitochondrial depolarization. Ten minutes after superfusion with PYY (1 $\mu \mathrm{M}$; first PYY panel $)$, both the electrical and mitochondrial responses were attenuated. Both properties increased 10 min later (second PYY panel), and both greatly increased at 15, 30, and 50 min after washout of PYY (bottom panels, respectively). Data are representative of seven similar experiments. Movies of the corresponding two-dimensional SD plots of the pre-, PYY, and recovery data are available in the electronic edition of this paper (http://www.jneurosci.org/supplemental/18/12/4570). 


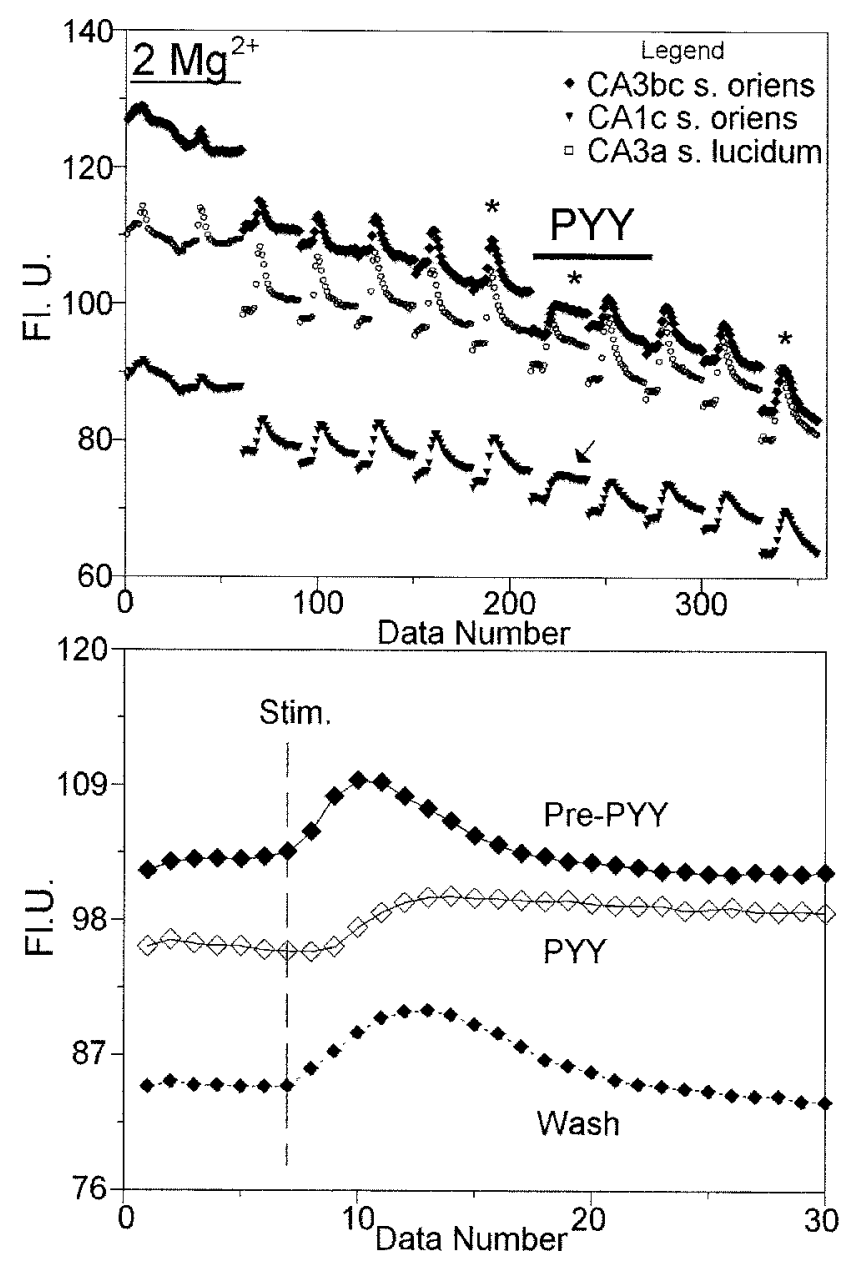

Figure 12. Raw fluorescence intensity plots for data shown in Figure 11. Top, Complete time course for fluorescence changes. Data from three regions (s.oriens near recording electrode, s.oriens in CA1c region, and s.luc. near stimulation electrode; see Fig. 11) are shown for each image collected. The $x$-axis indicates data number rather than time to facilitate display (experiment duration $=3.3 \mathrm{hr}$; each data point taken $\sim 5 \mathrm{sec}$ apart; data blocks are separated by $10-50 \mathrm{~min}$ ). Two stimulations in aCSF with $2 \mathrm{Mg}^{2+}$ produced large rises near the electrode and relatively little change in the s.oriens. The amplitude of the stimulation-evoked fluorescence increased at all sites during continued application of aCSF with 0.9 $\mathrm{mM} \mathrm{Mg}^{2+}$. PYY suppressed the increase and altered the kinetics of fluorescence changes (e.g., see arrow). After washout of PYY, the magnitude of the fluorescence changes continued to increase. Note the gradual, general loss of baseline fluorescence over the course of the experiment. Durations of treatments are indicated by horizontal bars. Bottom, The response for the $\mathrm{CA} 3 \mathrm{~b} / \mathrm{c}$ s.oriens region shown in greater detail for data sets indicated by the asterisks (top).

of $\left[\mathrm{Ca}^{2+}\right]_{\mathrm{i}}$ found at channel microdomains (e.g., Rizzuto et al., 1993) suggests that the mitochondrial contribution to $\mathrm{Ca}^{2+}$ signaling depends on the recent, local $\left[\mathrm{Ca}^{2+}\right]_{i}$ history. This mitochondrial "memory" of previous synaptic activity may be involved in shaping synaptic plasticity. Indeed, the proximity of mitochondria influences the activity of $\mathrm{Ca}^{2+}$ (Budd and Nicholls, 1996a), NMDA (Budd and Nicholls, 1996b), IP $_{3}$ (Jouaville et al., 1995; Simpson and Russell, 1996), and store-operated (Hoth et al., 1997) channels.

Aside from their routine, but important, roles as $\mathrm{Ca}^{2+}$ buffers and as metabolic energy sources, mitochondria may serve as modulators of gaseous messengers that affect synaptic activity. An increase in $\left[\mathrm{Ca}^{2+}\right]_{\mathrm{i}}$ and hence $\left[\mathrm{Ca}^{2+}\right]_{\mathrm{m}}$ has been shown to increase free radical production by mitochondria (Dykens, 1994; Dugan et al., 1995; Reynolds and Hastings, 1995; Bindokas et al., 1996) and by nitric oxide synthase (cf. Alagarsamy et al., 1994; Dawson and Snyder, 1994). Increased $\left[\mathrm{Ca}^{2+}\right]_{\mathrm{i}}$ also enables the generation of $\mathrm{O}_{2}{ }^{-}$from various other sources as well as the cascade of secondary reactive species (cf. Coyle and Puttfarcken, 1993). We found that glutamate-receptor agonists, as well as epileptiform activity, increased $\mathrm{O}_{2}{ }^{-}$production in slices (Bindokas et al., 1996; our unpublished observations). A direct consequence of this increase in mitochondrial free radical production will be alteration of redox-sensitive proteins as well as oxidative damage. The reaction of mitochondrially produced $\mathrm{O}_{2}{ }^{-}$with nitric oxide produces more reactive/toxic peroxynitrite $\left(\mathrm{ONOO}^{-}\right)$. Mitochondria may thus directly affect the balance of gaseous messengers. These messengers can reciprocally inhibit mitochondrial function at multiple sites (e.g., Castro et al., 1994; Hausladen and Fridovich, 1994; Schweizer and Richter, 1994; Cassina and Radi, 1996). Mitochondrial production of toxic radicals may also be involved in excitotoxic loss of synapses, and mitochondria may release apoptosis-inducing factors (cf. Kroemer et al., 1997; see also Ankarcrona et al., 1995). These events may underlie some of the damage seen in epilepsy.

\section{REFERENCES}

Alagarsamy S, Lonart G, Johnson KM (1994) Regulation of nitric oxide synthase activity in cortical slices by excitatory amino acids and calcium. J Neurosci Res 38:648-653.

Anderson WW, Lewis DV, Swartzwelder HS, Wilson WA (1986) Magnesium-free medium activates seizure-like events in the rat hippocampal slice. Brain Res 398:215-219.

Anderson WW, Swartzwelder HS, Wilson WA (1987) The NMDA receptor antagonist 2-amino-5-phosphonovalerate blocks stimulus traininduced epileptogenesis but not epileptiform bursting in the rat hippocampal slice. J Neurophysiol 57:1-21.

Andrew RD, MacVicar BA (1994) Imaging cell volume changes and neuronal excitation in the hippocampal slice. Neuroscience 62:371-383.

Ankarcrona M, Dypdukt JM, Bonfoco E, Zhivotovsky B, Orrenius S, Lipton SA, Nicotera P (1995) Glutamate-induced neuronal death: a succession of necrosis or apoptosis depending on mitochondrial function. Neuron 15:961-973.

Babcock DF, Herrington J, Goodwin PC, Park YB, Hille B (1997) Mitochondrial participation in the intracellular $\mathrm{Ca}^{2+}$ network. J Cell Biol 136:833-844.

Barish ME, Ichikawa M, Tominaga T, Matsumoto G, Iijima T (1996) Enhanced fast synaptic transmission and a delayed depolarization induced by transient potassium current blockade in rat hippocampal slice as studied by optical recording. J Neurosci 16:5672-5687.

Bernardi P, Petronilli V (1996) The permeability transition pore as a mitochondrial calcium release channel: a critical appraisal. J Bioenerg Biomembr 28:131-138.

Bindokas VP, Miller RJ (1995) Excitotoxic degeneration is initiated at non-random sites in cultured rat cerebellar neurons. J Neurosci 15:6999-7011.

Bindokas VP, Jordán J, Lee CC, Miller RJ (1996) Superoxide production in rat hippocampal neurons: selective monitoring by hydroethidine. J Neurosci 16:1324-1336.

Blasdel GG, Salama G (1986) Voltage-sensitive dyes reveal a modular organization in monkey striate cortex. Nature 321:579-585.

Bleakman D, Harrison NL, Colmers WF, Miller RJ (1992) Investigations into neuropeptide Y-mediated presynaptic inhibition in cultured hippocampal neurones of the rat. Br J Pharmacol 107:334-340.

Bragdon AC, Hisashi K, Wilson WA (1992) Suppression of interictal bursting in hippocampus unleashes seizures in entorhinal cortex: a proepileptic effect of lowering $\left[\mathrm{K}^{+}\right]_{\mathrm{o}}$ and raising $\left[\mathrm{Ca}^{2+}\right]_{\mathrm{o}}$. Brain Res 590:128-135.

Budd SL, Nicholls DG (1996a) A reevaluation of the role of mitochondria in neuronal $\mathrm{Ca}^{2+}$ homeostasis. J Neurochem 66:403-411.

Budd SL, Nicholls DG (1996b) Mitochondria, calcium regulation, and acute glutamate excitotoxicity in cultured cerebellar granule cells. J Neurochem 67:2282-2291. 
Cassina A, Radi R (1996) Differential inhibitory action of nitric oxide and peroxynitrite on mitochondrial electron transport. Arch Biochem Biophys 328:309-316.

Castro L, Rodriguez M, Radi R (1994) Aconitase is readily inactivated by peroxynitrite, but not by its precursor, nitric oxide. J Biol Chem 269:29409-29415.

Charles AC, Kodali SK, Tyndale RF (1996) Intercellular calcium waves in neurons. Mol Cell Neurosci 7:337-353.

Chervin RD, Pierce PA, Connors BW (1988) Periodicity and directionality of epileptiform discharges across neocortex. J Neurophysiol 60:1695-1713.

Colmers WF, Lukowiak KD, Pittman QJ (1987) Presynaptic action of neuropeptide $\mathrm{Y}$ in area CA1 of the rat hippocampal slice. J Physiol (Lond) 383:285-299.

Coyle JT, Puttfarcken P (1993) Oxidative stress, glutamate, and neurodegenerative disorders. Science 262:689-695.

Dani W, Chernjavsky A, Smith SJ (1992) Neuronal activity triggers calcium waves in hippocampal astrocyte networks. Neuron 8:429-440.

Dawson TM, Snyder SH (1994) Gases as biological messengers: nitric oxide and carbon monoxide in the brain. J Neurosci 14:5147-5159.

Denk W, Yuste R, Svoboda K, Tank DW (1996) Imaging calcium dynamics in dendritic spines. Curr Opin Neurobiol 6:372-378.

Duchen MR (1992) $\mathrm{Ca}^{2+}$-dependent changes in the mitochondrial energetics in single dissociated mouse sensory neurons. Biochem $\mathrm{J}$ 283:41-50.

Duchen MR, Biscoe TJ (1992) Relative mitochondrial membrane potential and $\left[\mathrm{Ca}^{2+}\right]_{\mathrm{i}}$ in type I cells isolated from the rabbit carotid body. J Physiol (Lond) 450:33-61.

Dugan LL, Sensi SL, Canzoniero LM, Handran SM, Rothman SM, Goldberg MP, Choi DW (1995) Mitochondrial production of reactive oxygen species in cortical neurons following exposure to $N$-methyl-Daspartate. J Neurosci 15:6377-6388.

Dykens JA (1994) Isolated cerebral and cerebellar mitochondria produce free radicals when exposed to elevated $\mathrm{Ca}^{2+}$ and $\mathrm{Na}^{+}$: implications for neurodegeneration. J Neurochem 63:584-591.

Ehrenberg B, Montana V, Wei M-D, Wuskell JP, Loew LM (1988) Membrane potential can be determined in individual cells from the Nernstian distribution of cationic dyes. Biophys J 53:785-794.

Emaus RK, Grunwald R, Lemasters JJ (1986) Rhodamine 123 as a probe of transmembrane potential in isolated rat-liver mitochondria: spectral and metabolic properties. Biochim Biophys Acta 850:436-448.

Erickson JC, Clegg KE, Palmiter RD (1996) Sensitivity to leptin and susceptibility to seizures of mice lacking neuropeptide Y. Nature 381:415-418.

Federico P, Borg SG, Salkauskus AG, MacVicar BA (1994) Mapping patterns of neuronal activity and seizure propagation by imaging intrinsic optical signals in the isolated whole brain of the guinea-pig. Neuroscience 58:461-480.

Gabso M, Neher E, Spira ME (1997) Low mobility of the $\mathrm{Ca}^{2+}$ buffers in axons of cultured Aplysia neurons. Neuron 18:473-481.

Ghosh A, Greenberg ME (1995) Calcium signaling in neurons: molecular mechanisms and cellular consequences. Science 268:239-247.

Gunter TE, Gunter KK, Sheu S-S, Gavin CE (1994) Mitochondrial calcium transport: physiological and pathological relevance. Am J Physiol 267:C313-C339.

Hajnóczky G, Robb-Gaspers LD, Seitz MB, Thomas AP (1995) Decoding of cytosolic calcium oscillations in the mitochondria. Cell $82: 415-424$.

Hassinger TD, Atkinson PB, Strecker GJ, Whalen LR, Dudek FE, Kossel AH, Kater SB (1995) Evidence for glutamate-mediated activation of hippocampal neurons by glial calcium waves. J Neurobiol 28:159-170.

Hausladen A, Fridovich I (1994) Superoxide and peroxynitrite inactivate aconitases, but nitric oxide does not. $J$ Biol Chem 269:29405-29408.

Hoth M, Fanger CM, Lewis RS (1997) Mitochondrial regulation of store-operated calcium signaling in $\mathrm{T}$ lymphocytes. $\mathrm{J}$ Cell Biol 137:633-648.

Hubel DH, Wiesel TN, Stryker MP (1978) Anatomical demonstration of orientation columns in macaque monkey. J Comp Neurol 177:361-380.

Ichas F, Jouaville LS, Mazat J-P (1997) Mitochondria are excitable organelles capable of generating and conveying electrical and calcium signals. Cell 89:1145-1153.

Iijima T, Witter MP, Ichikawa M, Tominaga T, Kajiwara R, Matsumoto G (1996) Entorhinal-hippocampal interactions revealed by real-time imaging. Science 272:1176-1179.
Jaffe LF (1993) Classes and mechanisms of calcium waves. Cell Calcium $14: 736-745$

Jaffe LF, Brown TH (1994) Metabotropic glutamate receptor activation induces calcium waves within hippocampal dendrites. J Neurophysiol $72: 471-474$.

Jouaville LS, Ichas F, Holmuhamedov EL, Camacho P, Lechleiter JD (1995) Synchronization of calcium waves by mitochondrial substrates in Xenopus laevis oocytes. Nature 377:438-441.

Khodorov B, Pinelis V, Vergun O, Storozhevykh T, Vinskaya N (1996) Mitochondrial deenergization underlies neuronal calcium overload following prolonged glutamate challenge. FEBS Lett 397:230-234.

Klapstein GJ, Colmers WF (1997) Neuropeptide Y suppresses epileptiform activity in rat hippocampus in vitro. J Neurophysiol 78:1651-1661.

Kroemer G, Zamzami N, Susin SA (1997) Mitochondrial control of apoptosis. Immunol Today 18:44-51.

Lipton P (1973) Effects of membrane depolarization on light scattering by cerebellar cortical slices. J Physiol (Lond) 231:365-383.

Loew LM, Carrington W, Tuft RA, Fay FS (1994) Physiological cytosolic $\mathrm{Ca}^{2+}$ transients evoke concurrent mitochondrial depolarizations. Proc Natl Acad Sci USA 91:12579-12583.

Lötscher H-R, Winterhalter KH, Carafoli E, Richter C (1980) The energy state of mitochondria during the transport of $\mathrm{Ca}^{2+}$. Eur J Biochem 110:211-216.

MacVicar BA, Hochman D (1991) Imaging of synaptically evoked intrinsic optical signals in hippocampal slices. J Neurosci 11:1458-1469.

MacVicar BA, Tse FW (1989) Local neuronal circuitry underlying cholinergic rhythmical slow activity in CA3 area of rat hippocampal slices. J Physiol (Lond) 417:197-212.

Magistretti PJ, Pellerin L (1996) Cellular bases of brain energy metabolism and their relevance to functional brain imaging: evidence for a prominent role of astrocytes. Cereb Cortex 6:50-61.

Mayer ML, Westbrook GL (1987) Permeation and block of $N$-methyl-Daspartate receptor channels by divalent cations in mouse cultured central neurones. J Physiol (Lond) 394:501-527.

McCormack JG, Halestrap AP, Denton RM (1990) Role of calcium ions in regulation of mammalian intramitochondrial metabolism. Physiol Rev 70:391-425.

Meldrum BS (1986) Cell damage in epilepsy and the role of calcium in cytotoxicity. Adv Neurol 44:849-855.

Miller LD, Petrozzino JJ, Golarai G, Connor JA (1996) $\mathrm{Ca}^{2+}$ release from intracellular stores induced by afferent stimulation of CA3 pyramidal neurons in hippocampal slices. J Neurophysiol 76:554-562.

Miller RJ (1991) The control of neuronal $\mathrm{Ca}^{2+}$ homeostasis. Prog Neurobiol 37:255-285.

Nafstad HJ, Blackstad TW (1966) Distribution of mitochondria in pyramidal cells and boutons in hippocampal cortex. Z Zellforsch Mikrosk Anat 73:234-245.

Nguyen PV, Atwood HL (1994) Altered impulse activity modifies synaptic physiology and mitochondria in crayfish phasic motor neurons. J Neurophysiol 72:2944-2955.

Nicholls D, Åkerman K (1982) Mitochondrial calcium transport. Biochim Biophys Acta 683:57-88.

Olney JW, Collins RC, Sloviter RS (1986) Excitotoxic mechanisms of epileptic brain damage. Adv Neurol 44:857-877.

Orbach HS, Cohen LB (1983) Optical monitoring of activity from many areas of the in vitro and in vivo salamander olfactory bulb: a new method for studying functional organization in the vertebrate central nervous system. J Neurosci 3:2251-2262.

Overly CC, Rieff HI, Hollenbeck PJ (1996) Organelle motility and metabolism in axons vs. dendrites of cultured hippocampal neurons. Cell Sci 109:971-980.

Parpura V, Basarsky TA, Liu F, Jeftinija S, Haydon PG (1994) Glutamate-mediated astrocyte-neuron signaling. Nature 369:744-747.

Petit J-M, Huet O, Gallet PF, Maftah A, Ratinaud M-H, Julien R (1994) Direct analysis and significance of cardiolipin transverse distribution in mitochondrial inner membranes. Eur J Biochem 220:871-879.

Porter JT, McCarthy KD (1996) Hippocampal astrocytes in situ respond to glutamate released from synaptic terminals. J Neurosci 16:5073-5081.

Qian J, Colmers WF, Saggau P (1997) Inhibition of synaptic transmission by neuropeptide $\mathrm{Y}$ in rat hippocampal area CA1: modulation of presynaptic $\mathrm{Ca}^{2+}$ entry. J Neurosci 17:8169-8177.

Reynolds IJ, Hastings TG (1995) Glutamate induces the production of reactive oxygen species in cultured forebrain neurons following NMDA receptor activation. J Neurosci 15:3318-3327. 
Rizzuto R, Brini M, Murgia M, Pozzan T (1993) Microdomains with high $\mathrm{Ca}^{2+}$ close to $\mathrm{IP}_{3}$-sensitive channels that are sensed by neighboring mitochondria. Science 262:744-747.

Schinder AF, Olson EC, Spitzer NC, Montal M (1996) Mitochondrial dysfunction is a primary event in glutamate neurotoxicity. J Neurosci 16:6125-6133.

Schweizer M, Richter C (1994) Nitric oxide potently and reversibly deenergizes mitochondria at low oxygen tension. Biochem Biophys Res Commun 204:169-175.

Senseman DM (1996) High-speed optical imaging of afferent flow through rat olfactory bulb slices: voltage-sensitive dye signals reveal periglomerular cell activity. J Neurosci 16:313-324.

Shoffner JM, Lott MT, Lezza AMS, Seibel P, Ballinger SW, Wallace DC (1990) Myoclonic epilepsy and ragged red fiber disease (MERRF) is associated with a mitochondrial DNA tRNA ${ }^{\text {Lys }}$ mutation. Cell 61:931-937.

Simpson PB, Russell JT (1996) Mitochondria support inositol 1,4,5triphosphate-mediated $\mathrm{Ca}^{2+}$ waves in cultured oligodendrocytes. J Biol Chem 271:33493-33501.

Sparagna GC, Gunter KK, Sheu S-S, Gunter TE (1994) Mitochondrial uptake from physiological-type pulses of calcium. J Biol Chem 270:27510-27515.

Stasheff SF, Bragdon AC, Wilson WA (1985) Induction of epileptiform activity in hippocampal slices by trains of electrical stimuli. Brain Res 344:296-302.

Stasheff SF, Anderson WW, Clark S, Wilson WA (1989) NMDA antagonists differentiate epileptogenesis from seizure expression in an in vitro model. Science 245:648-651.

Tang Y-G, Zucker RS (1997) Mitochondrial involvement in post-tetanic potentiation of synaptic transmission. Neuron 18:483-491.

Taylor GW, Merlin LR, Wong RKS (1995) Synchronized oscillations in hippocampal CA3 neurons induced by metabotropic glutamate receptor activation. J Neurosci 15:8039-8052.

Torbergsen T, Mathiesen E, Aasly J (1991) Epilepsy in a mitochondrial disorder. J Neurol Neurosurg Psychiatry 54:1073-1076.

Traub RD, Miles R (1991) Neuronal networks of the hippocampus. Cambridge, U.K.: Cambridge UP.

Tsacopoulos M, Magistretti PJ (1996) Metabolic coupling between glia and neurons. J Neurosci 16:877-885.

Ts'o DY, Frostig RD, Lieke EE, Grinvald A (1990) Functional organization of primate visual cortex revealed by high resolution optical imaging. Science 249:417-420.

Walther H, Lambert JD, Jones RS, Heinemann U, Hamon B (1986) Epileptiform activity in combined slices of hippocampus, subiculum and entorhinal cortex during perfusion with low magnesium medium. Neurosci Lett 69:156-161.

Westergaard N, Sonnewald U, Schousboe A (1994) Release of $\alpha$-ketoglutarate, malate and succinate from cultured astrocytes: possible role in amino acid neurotransmitter homeostasis. Neurosci Lett 176:105-109.

Westergaard N, Sonnewald U, Schousboe A (1995) Metabolic trafficking between neurons and astrocytes: the glutamate/glutamine cycle revisited. Dev Neurosci 17:203-211.

White RJ, Reynolds IJ (1996) Mitochondrial depolarization in glutamate-stimulated neurons: an early signal specific to excitotoxin exposure. J Neurosci 16:5688-5697.

Woldbye DPD, Madsen TM, Larsen PJ, Mikkelsen JD, Bolwig TG (1996) Neuropeptide Y inhibits hippocampal seizures and wet dog shakes. Brain Res 737:162-168.

Woldbye DPD, Larsen PJ, Mikkelsen JD, Klemp K, Madsen TM, Bolwig TG (1997) Powerful inhibition of kainic acid seizures by neuropeptide Y via Y5-like receptors. Nat Med 3:761-765. 